\title{
A Quantitative Analysis of the Retail Market for Illicit Drugs
}

\author{
Manolis Galenianos* $\quad$ Alessandro Gavazza ${ }^{\S}$
}

April 2014

\begin{abstract}
We develop a theoretical framework to study illicit drugs markets, and we estimate it using data on drug purchases. Buyers are searching for high-quality drugs, but they can determine drugs' quality (i.e., their purity) only after consuming them. Hence, sellers can rip-off first-time buyers, or can offer higher-quality drugs to induce buyers to purchase again from them. In equilibrium, a distribution of qualities persists. The estimated model implies that sellers' moral hazard reduces the average and increases the dispersion of drug purity, thereby affecting drug consumption. Moreover, the estimated model implies that increasing penalties may increase the purity and the affordability of drugs traded, because it increases sellers' relative profitability of targeting loyal buyers versus first-time buyers.
\end{abstract}

\section{PRELIMINARY AND INCOMPLETE}

*Department of Economics, Royal Holloway, University of London. Egham Hill, Egham TW20 0EX, United Kingdom. Email: manolis.galenianos@gmail.com.

$\S$ Department of Economics, London School of Economics. Houghton Street, London WC2A 2AE, United Kingdom. Email: a.gavazza@lse.ac.uk. 


\section{Introduction}

How do markets for illicit commodities, such as narcotics, differ from regular markets? What would happen to the consumption and prices of narcotics if their trade were legalized? How do changes in the intensity of enforcement affect them?

We seek to understand these issues by building and estimating a model that focuses on pervasive sellers' moral hazard as the distinguishing characteristic of the market for illicit drugs (i.e., the "cutting" of drugs). ${ }^{1}$ We quantify the effects of sellers' moral hazard on drugs' pure-gram prices and drugs' consumption, possibly providing some insights on how market outcomes would differ if this market were legal. The presence of moral hazard leads to counter-intuitive effects of policing, as well.

We model a market in which buyers with heterogeneous willingness to pay for drugs search for sellers with heterogeneous costs of supplying drugs. Following the key insight of Galenianos, Pacula and Persico (2012), buyers cannot observe drug purity before consuming it - i.e., illicit drugs are credence goods; this is one key way in which the model captures an illegal market, in which quality is non-contractible and no institutions can enforce quality standards. Buyers' inability to ascertain quality creates a trade-off for sellers. On one hand, they can offer zero-purity drugs to first-time buyers, thereby maximizing instantaneous profit. On the other hand, they can offer higher-quality drugs that induce buyers to purchase again from them, thereby increasing their customer base. In equilibrium, a distribution of quality levels persist: high-cost sellers choose to cheat and rip-offs their (first-time) buyers, whereas low-cost sellers offer positive purity levels, with the lowest-cost sellers offering the purest drugs.

In our quantitative analysis, we estimate the model combining three distinct datasets that provide three key pieces of information on the powder cocaine market: 1) the distribution of drug qualities traded in the market; 2) how frequently buyers purchase drugs; and 3) whether buyers purchased drugs from their regular, long-term sellers. Overall, the model fits the data well. Moreover, the estimates imply that sellers' profits are extremely skewed, with very few (low-cost) sellers reaping substantial profits, whereas most sellers earn less than the minimum wage, in agreement with the descriptive evidence reported by Levitt and Venkatesh (2000).

We use our parameterized model to perform counterfactual analyses. Specifically, the

\footnotetext{
${ }^{1}$ For instance, a significant proportion of drug purchases - usually 5-10\% - involve zero purity level, i.e. are complete rip-offs. It is hard to find a legal market with comparable levels of outright fraud.
} 

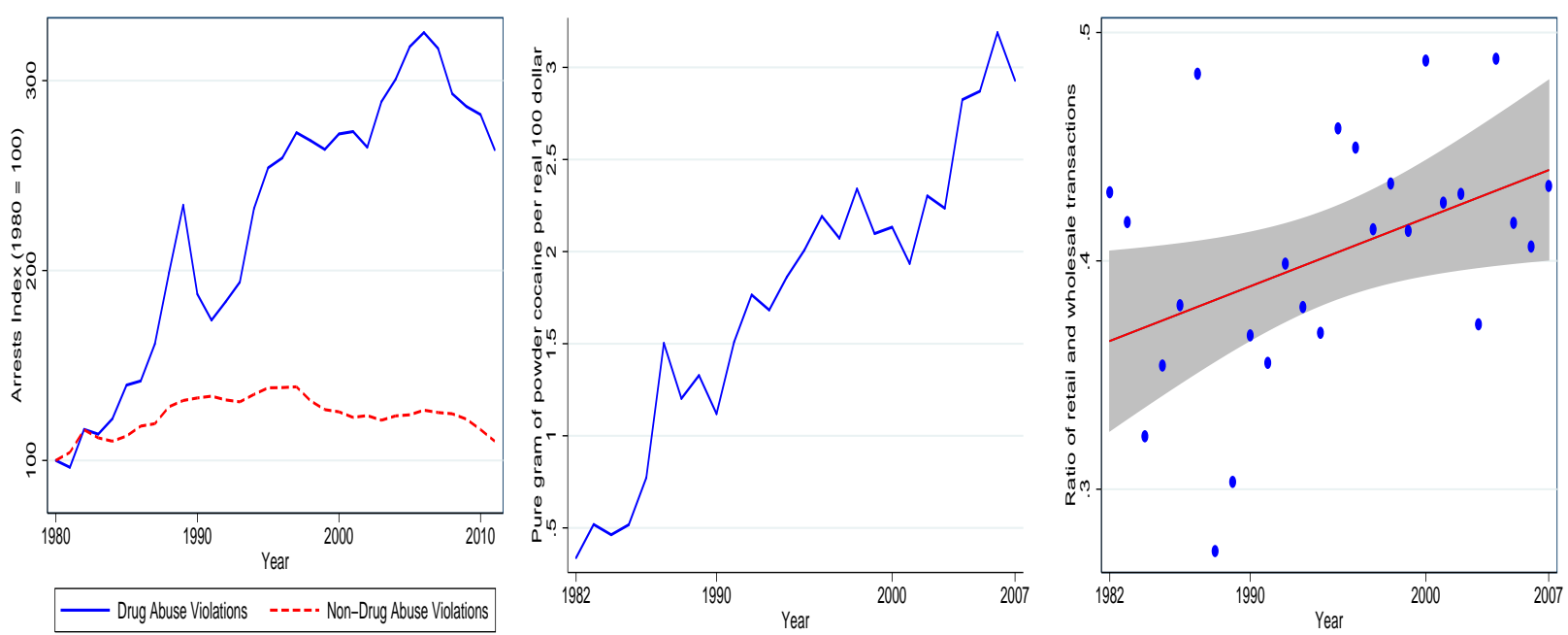

FIG. 1: The left panel displays the number of arrests in the United States in the years 1980-2010, relative to the year 1980 . The middle panel displays the average pure gram of powder cocaine per 100 real dollars, retail transactions 1982-2007 (the base year is 1983). The right panel displays the ratio between annual average pure grams of powder cocaine per 100 real dollars in retail transactions and annual average pure grams of powder cocaine per 100 real dollars in wholesale transactions, years 1982-2007.

model allows us to quantify the role of sellers' moral hazard due to buyers' inability to verify the quality of drugs. This type of buyers' imperfect information is one key characteristic of illegal markets because, in a legal market, the quality of the product is more-easily verifiable and contractible. Our counterfactual analysis quantifies the effect of this information friction on market outcomes, possibly providing some insights on how outcomes would differ if the market were legal, with buyers having better information about product quality before trading. This counterfactual analysis reveals that zero-purity drugs disappear from the market. Moreover, sellers have to increase quality to induce their first-time occasional buyers to purchase and, possibly, to become their loyal customers and, thus, the average purity increases by approximately 9 percent and the variance of purity decreases by approximately 50 percent. Hence, a larger fraction of buyers is matched to a regular seller, thereby affecting buyers' drug consumption.

We further use our model to study the role of differential penalties on retail buyers and on retail sellers. This is interesting for at least two reasons. First, the U.S. have markedly increased the enforcement and severity of drug laws, the so-called "war on drugs." The left panel of Figure 1 displays one of the most visible outcomes of this policy: the number of people arrested for drug-related offenses has tripled, whereas the number of arrests for non- 
drug related offenses has barely changed over the same period. At the same time, the middle panel of Figure 1 displays that drugs have become dramatically cheaper: the amount of pure cocaine that can be purchased with 100 real dollars has increased sixfold over since the early 1980s. Most notably, the right panel of Figure 1 shows that the most significant reduction in drugs' real prices has occurred at the retail level rather than at the wholesale level (of value up to $\$ 200$ or above $\$ 2000$, respectively), motivating this paper's focus on retail markets. Second, countries have mild or no penalties on illicit drugs' buyers and strong penalties on drugs' sellers, whereas the United States enforce strict penalties on both buyers and sellers. We find that increasing enforcement on sellers leads to an increase in the average quality offered in the market, thereby making drugs more affordable. The reason is that higher penalties decrease the number of sellers, thus making it more difficult for buyers to meet sellers. In a market with moral hazard, this lower meeting rate decreases sellers' incentives to make quick profits by selling zero-purity drugs. Instead, sellers increase the qualities of drugs to attract loyal-buyers. More generally, the counterfactual analyses highlight that long-term relationships are more valuable in a market with less frequent search. Thus, to the extent that an increase in police enforcement reduces the intensity of search in the market, it helps strengthen the long-term relationships that help overcome the inherent moral hazard problem in an illegal market and, therefore, leads to greater average quality.

\section{Data}

We combine three distinct datasets. The first is an extensive database on drug purchases. The second is a survey that collects information about drug use among those committing crimes. The third is a survey that collects information about drug use among the noninstitutionalized population aged 12 and older. We now describe each dataset in more detail.

STRIDE - The System to Retrieve Information from Drug Evidence (STRIDE) is a database of drug exhibits sent to Drug Enforcement Administration (DEA) laboratories for analysis. Exhibits in the database are from the DEA, other federal agencies, and local law enforcement agencies. The data contain records of acquisitions of illegal drugs by undercover agents and informants of the DEA. These data are widely used in economic analyses of markets for illegal drugs, although STRIDE is not a representative sample of drugs available 
in the United States. ${ }^{2}$

The entire dataset has a total of approximately 915,000 observations for the period 19822007 for a number of different drugs and acquisition methods. We focus on powder cocaine and keep the observations acquired through purchases (i.e., we drop seizures) and clean the data of missing values and other unreliable observations, as suggested by Arkes et al. (2004). While we use the STRIDE data to present trends for our entire sample period, we will restrict our quantitative analysis of Section 4 to the years 2001-2003 because of the time limitations of our other data sources, as described below. Moreover, since the focus of our model is on retail transactions, we include in our estimation sample only purchases with a value of less than $\$ 200$ in real 1983 dollars.

ADAM - The Arrestee Drug Abuse Monitoring (ADAM) data set is a quarterly survey of persons arrested or booked on local and state charges within the past 48 hours in various ADAM metropolitan areas in the United States. ${ }^{3}$ Individuals involved in non-drug and drug-related crimes are interviewed about the use, importance and role of drugs and alcohol. The arrestees participated in the survey voluntarily under full confidentiality. ${ }^{4}$ In addition to interviewing arrestees, urine samples are requested and analyzed for validation of selfreported drug use. Since 2000, a drug market procurement module has been included as part of the quarterly survey and collects information on the arrestee's most recent drugs purchase for all arrestees who report having used drugs in the previous 30 days. Information collected includes number of drug purchases in the past 30 days, number of drug dealers they transacted with, whether they last purchased from their regular dealer, and whether the arrestee experienced any difficulty in locating a dealer or buying the drug. We have data from the 2001-2003 surveys.

\footnotetext{
${ }^{2}$ The reliability of the STRIDE data set has been called into question by Horowitz (2001), who remarked that depending on which agency collected the data (DEA or other law enforcement agency), the time series of drug prices in Washington, D.C. look somewhat different. However, Arkes et al. (2008) show that the inconsistencies identified by Horowitz (2001) largely disappear simply by controlling for the size of the transaction (above or below 5 grams) when combined with other data cleaning issues raised by Horowitz (2001). Mindful of this finding, we are careful to restrict our analysis to the relatively narrow sample of transactions whose value is below 100 constant 1983 dollars. Also, Arkes et al. (2008) show that the price series for different drugs obtained from STRIDE predict, in a Granger sense, the number of drugrelated admissions to emergency rooms (DAWN data set). Overall, we feel that Arkes et al. (2008) make a compelling case for the usefulness of the STRIDE dataset when used carefully, i.e., without aggregating across transactions of vastly different sizes.

${ }^{3}$ The number of these areas changes from years to year based on the availability of the data. From 2001 to 2003 , it has been 33,36 and 39 , respectively.

${ }^{4}$ Dave (2007) notes that only about $10 \%$ of the arrestees reject the interview request.
} 


\section{TABLE 1: Summary statistics}

\begin{tabular}{lcccc}
\hline \hline Panel A: Stride & Obs. & Mean & St. Dev. & Median \\
\hline Price (2003 dollars) & 417 & 108.128 & 50.718 & 104.222 \\
Weight (Grams) & 417 & 1.885 & 1.569 & 1.5 \\
Potency (\%) & 417 & 55.346 & 25.921 & 61 \\
Pure Quantity & 417 & 1.000 & 0.897 & 0.800 \\
Pure Grams Per \$100 & 417 & 3.267 & 2.408 & 2.846 \\
\hline Panel B: ADAM & & & & \\
\hline Any Purchase in Past 30 Days & 14,627 & 0.332 & 0.470 & 0 \\
-Purchases in Past 30 Days & 4,857 & 7.392 & 8.904 & 3 \\
-Purchased From Regular Dealer & 4,320 & 0.615 & 0.486 & 1 \\
\hline Panel C: NSDUH & & & & \\
\hline Consumed Cocaine LaSt Year & 164,870 & 0.033 & 0.178 & 0 \\
-Cocaine Frequency Past Month & 1,853 & 4.957 & 6.270 & 2 \\
\hline \hline
\end{tabular}

Notes - This table provides summary statistics of the variables used in the empirical analysis. Panel A presents summary statistics of the variables obtained from the STRIDE dataset; Panel B presents summary statistics of the variables obtained from the ADAM dataset; and Panel C presents summary statistics of the variables obtained from the NSDUH dataset. Drug prices have been deflated using the GDP Implicit Price Deflator, with 2003 as the base year.

NSDUH - The National Survey on Drug Use and Health (NSDUH) is an annual nationwide survey involving interviews with approximately 70,000 randomly-selected non-institutionalized individuals aged 12 and older with the goal of providing national data on the use of tobacco, alcohol, illicit drugs (including non-medical use of prescription drugs) and mental health in the United States. The survey asks questions on individuals' consumptions of several illicit drugs, including the frequency of use of during the previous month. We use the data for cocaine consumption in the years 2001-2003.

\subsection{Data Description}

Table 1 provides summary statistics of the main variables used in the quantitative analysis. Panel A refers to the STRIDE Dataset, Panel B to the ADAM dataset, and Panel C to the NSDUH dataset.

Panel A reports some interesting patterns. While the transactions display some het- 


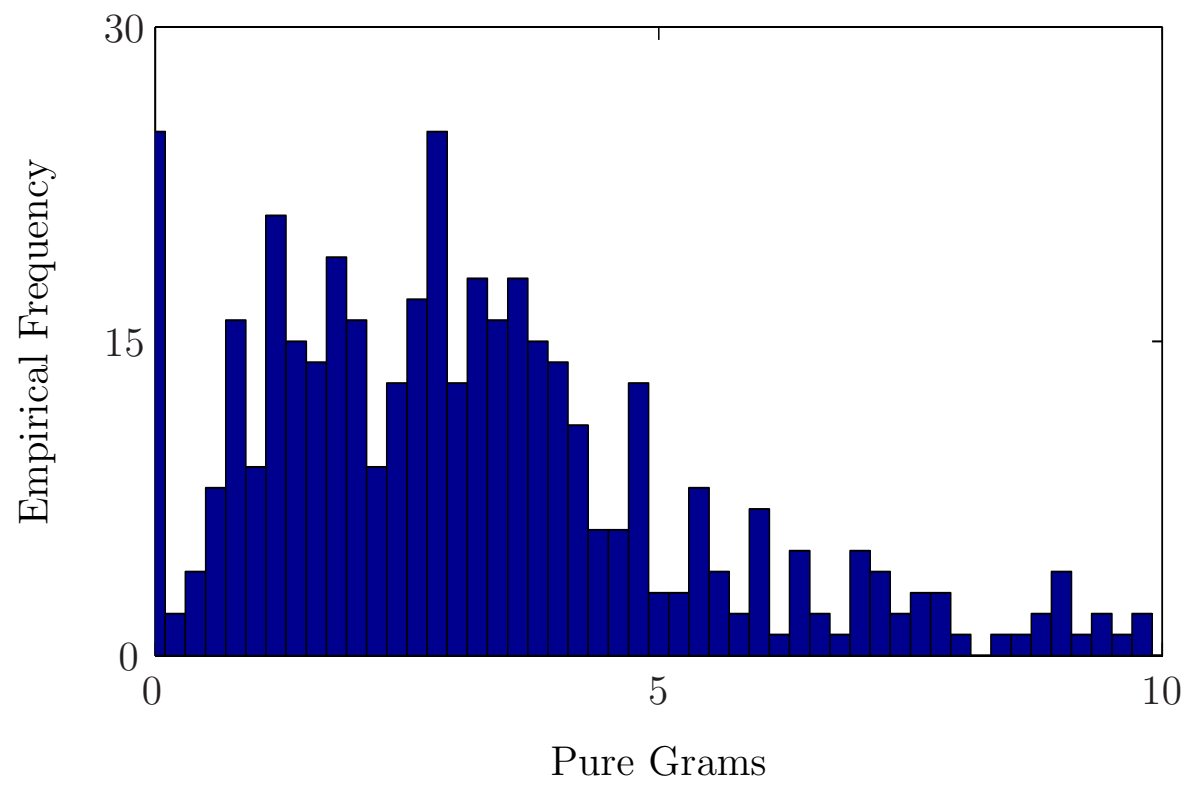

FIG. 2: Histogram of pure grams per $\$ 100$.

erogeneity in their dollar values, the heterogeneity of PURE QUANTITY (the product of Weight and Potency) is substantially larger. We take the ratio of PURE QuAntity and PRICE to construct the variable PURE GRAMS PER \$100; figure 2 displays its empirical distribution, which displays substantial variation, with 6 percent of the observations having a value of zero-i.e., complete ripoffs.

Panel B reports that one-third of all arrestees purchased cocaine in the past 30 days. Of those who purchased cocaine, the average number of PURCHASES IN PAST 30 DAYs equals 7.39. (Thus, the unconditional average of Purchases In PAst 30 Days is 2.45.) Of those who purchased cocaine, 61 percent report consuming from their regular source. Interestingly, individuals purchasing from their regular dealers report an average of 9.11 PURCHASES IN PAST 30 DAYs, whereas individuals purchasing either from an occasional source or from a new source have an average of 5.51 Purchases in PAst 30 Days. We interpret this difference as different consumption rates between buyers who are currently matched to a seller and buyers who are currently not matched.

Panel C reports that 3.3 percent of the U.S. non-institutionalized population aged 12 and older reports consuming cocaine in the previous year, corresponding to approximately four million people; we take this as the number of active buyers in the market. Moreover, individuals who are reporting consuming cocaine in the previous month consumed approximately five times in the month. Notice that this consumption frequency is lower than in the 
ADAM dataset, suggesting that heavy-users are over-represented in ADAM.

Overall, these three datasets provide a rich description of the retail cocaine market and are well-suited to investigating the importance of search frictions and the role of buyer-seller relationships. Specifically, our model interprets the dispersion of PURE GRAMS PER \$100 as departure of the law-of-one-price originating from search frictions. Moreover, the NSDUH data allow us to obtain an estimate of the number of active buyers and their consumption frequencies. Further, the NSDUH dataset also allows us to reweight the observations in the ADAM datasets. The ADAM dataset is also useful to measure the frequency and duration of buyer-seller long-term relationships.

\section{Model}

Time runs continuously, the horizon is infinite and the future is discounted at rate $r$.

There is a continuum of potential buyers of measure $\bar{B}$ who are heterogeneous with respect to their preferences for consuming drugs. A buyer's marginal utility of consuming drugs is denoted by $z$ and is distributed according to a continuous and connected distribution $\tilde{M}(\cdot)$ with support $[0, \bar{z}]$. Each buyer decides whether to participate the market. If he does not participate, his payoff is zero. If he participates, he pays entry cost $K_{B}$, which represents the possibility of arrest, ${ }^{5}$ and he meets with sellers. The measure of buyers who participate in the market is denoted by $B$ and the distribution of their types is denoted by $M(\cdot)$. At any point in time, a buyer is either unmatched or matched with a seller (his "regular" seller). Buyers maximize their expected discounted utility.

There is a continuum of potential sellers of measure $\bar{S}$ who are ex ante identical. A seller decides whether to pay entry cost $K_{S}$ and participate in the market. If he participates, he draws his cost $c$ from distribution $D(\cdot)$ which is continuous and connected with support $(0, \infty) .{ }^{6}$ The measure of sellers who participate in the market is denoted by $S$. Sellers maximize their discounted steady state profits.

Buyers and sellers want to trade with each other. There are two types of meetings between buyers and sellers: "new" meetings, where a buyer and a seller meet for the first time, and "repeat" meetings, where a buyer meets his regular seller. At a meeting, a transaction takes place and is followed by the transition between the matched and unmatched state.

\footnotetext{
${ }^{5}$ This cost can also be formulated as a flow cost without changing any of the results.

${ }^{6}$ The information structure (described below) means that it will be trivially optimal for a seller to stay in the market once he has paid the entry cost.
} 
In a transaction the buyer pays a fixed price $p$ and receives quality $q$. At the time of the transaction, both buyer and seller observe $p$ but the quality $q$ fetched by $p$ cannot be determined by the buyer. After the transaction, the buyer consumes the good and the quality of the purchase is perfectly revealed. The instantaneous utility that a type- $z$ buyer receives from consuming quality $q$ is equal to $z q$. The quality $q$ is chosen by the seller at cost $c q$.

The main assumption on sellers' behavior is that, once they decide on the quality level that they offer, they commit to their decision forever. That is, a seller supplies the same quality at all times and, as a result, the buyer knows the quality that he will receive from a particular seller once he has sampled from him. Let $F(\cdot)$ denote the distribution of qualities offered in the market.

After the transaction, the buyer decides whether to match with that seller. Specifically, an unmatched buyer chooses whether to to remain unmatched or to match with the seller; a matched buyer chooses whether to return to his previous regular seller or to match with the new seller, thereby severing his earlier match. In addition to this endogenous match dissolution, a match between a buyer and a seller is exogenously destroyed at rate $\delta$ and in this event the buyer becomes unmatched.

The flow of new meetings is determined by a matching function $m(B, S)$. The matching function is increasing and concave in both arguments, and satisfies $m(0, S)=m(B, 0)=0$ and the Inada conditions. Let $\theta=\frac{B}{S}$, and denote the rate at which a buyer meets with a new seller by $\alpha_{B}(\theta)$ and the rate at which a seller meets with a new buyer by $\alpha_{S}(\theta)$ :

$$
\begin{aligned}
\alpha_{B}(\theta) & =\frac{m(B, S)}{B}, \\
\alpha_{S}(\theta) & =\frac{m(B, S)}{S} .
\end{aligned}
$$

Note that $\alpha_{B}(\cdot)$ is decreasing and $\alpha_{S}(\cdot)$ is increasing in the buyer-seller ratio $\theta$.

The flow of repeat meetings is equal to $\phi$, which is the rate that a matched buyer contacts his seller. Notice that matched buyers might also participate in new meetings, i.e. they meet new sellers.

A potential buyer decides whether to participate in the market. Denote the value of an unmatched buyer of type $z$ who participates in the market by $\bar{V}_{z}$. A buyer of type $z$ compares the costs and benefits of entering the market and he participates if and only if

$$
r \bar{V}_{z} \geq K_{B}
$$


A participating buyer chooses the reservation quality for becoming matched with a new seller, as a function of whether he is currently matched or unmatched. The reservation value of a matched buyer is, trivially, given by the quality that he receives from his regular seller. Let $R_{z}$ denote the reservation quality of an unmatched buyer of type $z$ and let $H(\cdot)$ denote the distribution of unmatched buyers' reservation qualities.

A potential seller chooses whether to participate in the market and, if so, he chooses the quality level $q$ that maximizes his steady state profits after observing his cost $c$. Steady state profits have two components: the margin per transaction and the steady state flow of transactions. The profit margin from each transaction is equal to $p-c q$. The flow of transactions is $t(q)=t_{N}+t_{L}(q)$ where $t_{N}$ refers to new buyers and $t_{L}(q)$ refers to loyal buyers. Steady state profits are

$$
\pi_{c}(q)=(p-c q)\left(t_{N}+t_{L}(q)\right)
$$

Thus, sellers' free entry condition implies that sellers' expected profits equal their entry cost:

$$
\int_{0}^{\infty} \pi_{c}(q) d D(c)=K_{S}
$$

We are now ready to define the equilibrium.

Definition 1 An equilibrium is the actions of buyers $M(\cdot), H(\cdot), B$ and the actions of sellers $F(\cdot), S$ such that all agents optimize.

\subsection{The Buyers}

We derive the buyers' optimal action, taking as given the distribution of offered qualities $F(\cdot)$ and the number of participating sellers $S$. Denote the average quality of a new draw by $\hat{q}=\int_{0}^{\bar{q}} x d F(x)$. Proposition 2 summarizes this Section's results.

Proposition 2 Given $F(\cdot)$ and $S$ :

1. If $\frac{p}{\hat{q}} \geq \bar{z}$ then there is no buyer entry: $B=0$.

2. If $\frac{p}{\hat{q}}<\bar{z}$ then there is a unique buyer type $z^{*} \leq \bar{z}$ such that all buyers with $z>z^{*}$ participate in the market and all buyers with $z \leq z^{*}$ do not. 
3. The measure of buyers in the market is $B=\bar{B}\left(1-\tilde{M}\left(z^{*}\right)\right)$ and the distribution of their types in the market is given by

$$
M(z)= \begin{cases}0 & \text { if } z \leq z^{*} \\ \frac{\tilde{M}(z)-\tilde{M}\left(z^{*}\right)}{1-\tilde{M}\left(z^{*}\right)} & \text { if } z \geq z^{*}\end{cases}
$$

4. The marginal buyer type is given by the solution to:

$$
\alpha_{B}(\theta)\left(z^{*} \int_{0}^{\bar{q}} x d F(x)+z^{*} \int_{p / z^{*}}^{\bar{q}} \frac{\phi(1-F(x))}{r+\delta+\alpha_{B}(\theta)(1-F(x))} d x-p\right)=K_{B}
$$

5. The reservation quality of a type-z buyer who participates in the market is $R_{z}=\frac{p}{z}$ and the distribution of reservation qualities in the market is

$$
H(R)= \begin{cases}0 & \text { if } R \leq \underline{R} \\ \frac{1-M\left(\frac{p}{R}\right)}{1-M\left(z^{*}\right)} & \text { if } R \in[\underline{R}, \bar{R}] \\ 1 & \text { if } R \geq \bar{R}\end{cases}
$$

where $\underline{R}=R_{z}=\frac{p}{z}$ and $\bar{R}=R_{z^{*}}=\frac{p}{z^{*}}$.

To prove the Proposition we proceed in three steps. First, we examine the behavior of a type- $z$ buyer who has entered the market to determine his reservation quality $R_{z}$. Second, we examine an individual buyer's participation decision as a function of the actions of sellers and other buyers, $F(\cdot)$ and $\theta$. Third, we aggregate the decisions of all buyers, to derive the measure of buyers who participate and the distribution of their types.

The reservation quality for an unmatched buyer can be found by equating the value of remaining unmatched with the value of becoming matched. The value functions of being unmatched or matched with a seller who offers $q$ for a buyer of type $z$ are given by:

$$
\begin{aligned}
r \bar{V}_{z} & =\alpha_{B}(\theta)\left(z \int_{0}^{\bar{q}} x d F(x)+\int_{R_{z}}^{\bar{q}}\left(V_{z}(x)-\bar{V}_{z}\right) d F(x)-p\right) \\
r V_{z}(q) & \left.=\phi(z q-p)+\alpha_{B}(\theta)\left(z \int_{0}^{\bar{q}} x d F(x)+\int_{q}^{\bar{q}}\left(V_{z}(x)-V_{z}(q)\right) d F(x)-p\right)+\delta\left(\bar{V}_{z}-V_{z}(q) 3\right)\right)
\end{aligned}
$$


Equating (2) with (3) yields:

$$
\bar{V}_{z}=V_{z}\left(R_{z}\right)
$$

which implies:

$$
R_{z}=\frac{p}{z} .
$$

This proves the first half of part 2 of Proposition 2.

Two features are worth commenting on. First, the reservation quality does not depend on the distribution of offered qualities, $F(\cdot)$. This is due to the fact that the arrival rate of new sellers is the same when matched and unmatched. Therefore to the extent that a buyer's utility is greater than the price, there is no further opportunity cost to forming a match. ${ }^{7}$ Second, the reservation quality is decreasing in buyers' marginal utility. This feature is due to the fact that, conditional on $q$, the gains from trade are higher when the buyer has greater marginal utility of consumption and therefore his reservation quality is lower than that of a low- $z$ buyer.

An individual buyer takes as given the actions of sellers $\{F(\cdot), S\}$ and other buyers $(B)$ and decides whether to participate in the market. The actions of other agents are summarized as $\{F(\cdot), \theta\}$. To examine the individual buyer's choice, we write his value of participating $\bar{V}_{z}$ in a more convenient way.

Using integration by parts, the value of being unmatched satisfies:

$$
r \bar{V}_{z}=\alpha_{B}(\theta)\left(z \int_{0}^{\bar{q}} q d F(q)+\int_{R_{z}}^{\bar{q}} V_{z}^{\prime}(q)(1-F(x)) d x-p\right) .
$$

Moreover, differentiating equation (3) with respect to $q$, we obtain

$$
V_{z}^{\prime}(q)=\frac{\phi z}{r+\delta+\alpha_{B}(\theta)(1-F(q))} .
$$

Thus, we can combine the previous two equations:

$$
r \bar{V}_{z}=\alpha_{B}(\theta)\left(z \hat{q}+z \int_{\frac{p}{z}}^{\bar{q}} \frac{\phi(1-F(x))}{r+\delta+\alpha_{B}(\theta)(1-F(x))} d x-p\right) .
$$

\footnotetext{
${ }^{7}$ In contrast, in GPP a matched buyer only meets new sellers when his regular seller is unavailable and matching with a seller reduces the chance of meeting a better seller later on. The magnitude of that opportunity cost depends on the distribution of offered qualities which is why in GPP the reservation quality depends on the distribution of offered qualities.
} 
We now determine whether a buyer of type $z$ participates in the market. Consider the limit where there are very few buyers per seller $(\theta \rightarrow 0)$ and note that:

$$
\lim _{\theta \rightarrow 0} r \bar{V}_{z}=\lim _{\theta \rightarrow 0} \alpha_{B}(\theta)(z \hat{q}-p)
$$

and

$$
\lim _{\theta \rightarrow 0} \alpha_{B}(\theta)(z \hat{q}-p) \geq K_{B} \Leftrightarrow z>\frac{p}{\hat{q}}
$$

Therefore a buyer with $z>\frac{p}{\hat{q}}$ might enter if the arrival rate of new meetings is high enough and does not enter if $z \leq \frac{p}{\hat{q}}$ regardless of $\theta$. As a corollary, if $\bar{z} \leq \frac{p}{\hat{q}}$ then no buyer enters, proving part 1 of Proposition 2.

Furthermore, a buyer's value of participating in the market is strictly decreasing in $\theta$, i.e. it is increasing in the rate of meeting with sellers:

$$
\frac{\partial r \bar{V}_{z}}{\partial \theta}=\alpha_{B}^{\prime}(\theta)(z \hat{q}-p)+\frac{z \alpha_{B}^{\prime}(\theta)(r+\delta)}{\alpha_{B}(\theta)^{2}} \int_{\frac{p}{z}}^{\bar{q}} \frac{\phi(1-F(q))}{\left(\frac{r+\delta}{\alpha_{B}(\theta)}+1-F(q)\right)^{2}} d q<0 .
$$

In the limit, if a buyer never meets with sellers, then he does not enter:

$$
\lim _{\theta \rightarrow \infty} r \bar{V}_{z}=0<K_{B}
$$

Therefore, for each buyer of type $z$ with $z>\frac{p}{\hat{q}}$ there is a unique $\theta(z)$ such that he participates if $\theta \leq \theta(z)$ and stays out otherwise.

The value of participating in the market is, unsurprisingly, negative for buyers who receive no utility from consuming and is strictly increasing in a buyer's marginal utility of consumption:

$$
\begin{aligned}
r \bar{V}_{0} & =-\alpha_{B}(\theta) p<0 \\
\frac{\partial r \bar{V}_{z}}{\partial z} & =\alpha_{B}(\theta) \int_{0}^{\bar{q}} q d F(q)+\alpha_{B}(\theta) \int_{R_{z}}^{\bar{q}} \frac{\phi(1-F(q))}{r+\delta+\alpha_{B}(\theta)(1-F(q))} d q+\frac{p}{z^{2}} \frac{\alpha_{B}(\theta) \phi\left(1-F\left(R_{z}\right)\right)}{r+\delta+\alpha_{B}(\theta)\left(1-F\left(R_{z}\right)\right)}>0
\end{aligned}
$$

Taking $\theta$ as given, there is a unique $z(\theta)$ such that a buyer participates if $z \geq z(\theta)$ and does not participate otherwise.

We now prove that $z^{*}$ is unique, taking into account that the number of buyers depends on $z^{*}$ according to $B=\bar{B}\left(1-\tilde{M}\left(z^{*}\right)\right)$. First, note that when $z^{*}=0$ we have $r \bar{V}_{z^{*}}<K_{B}$. 
Furthermore, when $z^{*}=\bar{z}$ we have $r \bar{V}_{\bar{z}}>K_{B}$, assuming of course that $\bar{z}>\frac{p}{\hat{q}}$, because otherwise no buyers enter.

To prove that the uniqueness of $z^{*}$ we need to show that the value of the marginal type is increasing in his own type. The unmatched value of the marginal buyer depends on $z^{*}$ as follows:

$$
\frac{d r \bar{V}_{z^{*}}}{d z^{*}}=\frac{\partial r \bar{V}_{z^{*}}}{\partial z^{*}}+\frac{\partial r \bar{V}_{z^{*}}}{\partial \theta}\left(-\bar{B} \tilde{M}^{\prime}\left(z^{*}\right)\right)>0 .
$$

Therefore, there is a unique $z^{*}$ such that the unmatched value of the marginal buyer is exactly equal to $K_{B}$ and it is defined by equation (1). This completes the proof of Proposition 2, parts 2,3 and 4 .

Finally, let $z(R)$ denote the buyer type whose reservation quality is equal to $R$. Rearranging equation (4) we have:

$$
z(R)=\frac{p}{R}
$$

Furthermore, note that $R_{z(R)}=R$ and $z \leq z(R) \Leftrightarrow R_{z} \geq R$. Given $z^{*}$, the equilibrium distribution of reservation qualities mirrors the distribution of marginal utilities according to Proposition 2, part 5.

This completes the characterization of buyers' behavior.

\subsection{The Sellers}

We derive the sellers' profits and describe their actions, taking as given the measure of buyers who participate $B$ and the distribution of reservation qualities $H(\cdot)$. The distribution of buyer types does not affect sellers over and above the distribution of reservation qualities.

A measure $S$ of sellers participate in the market, which is determined through free entry. Each seller draws the marginal cost $c$ of providing a unit of quality from some distribution $D(\cdot)$. The problem of a seller of type $c$ is to choose a level of quality $\hat{q}(c)$ that maximizes his steady state profits. Steady state profits have two components: the margin per transaction and the steady state flow of transactions. The profit margin from each transaction is equal to $p-c q$. The flow of transactions is $t(q)=t_{N}+t_{L}(q)$ where $t_{N}$ refers to new buyers and $t_{L}(q)$ refers to loyal buyers. Steady state profits are:

$$
\pi_{c}(q)=(p-c q)\left(t_{N}+t_{L}(q)\right) .
$$


We first derive some necessary conditions on the distribution of offered qualities.

Lemma 3 In equilibrium, the quality distribution $F$ :

1. has support on a subset of $\{0\} \cup[\underline{q}, \bar{q}]$,

2. $\underline{q} \in[\underline{R}, \bar{R}]$,

3. is continuous on $[0, \bar{q}]$.

Proof. For $q \in[0, \underline{R})$ we have $t(q)=t_{N}$ which implies that $\pi_{c}(0)>\pi_{c}(q)$ for $q \in(0, \underline{R})$. Therefore either $q=0$ or $q \geq \underline{q}$ for some $\underline{q} \geq \underline{R}$. If $\underline{q}>\bar{R}$ then $t(q)=t(\bar{R})$ for $q \in[\bar{R}, \underline{q}]$ which implies that $\pi_{c}(\bar{R})>\pi_{c}(q)$ for $q \in(\bar{R}, \underline{q}$. Therefore, $\underline{q} \leq \bar{R}$. The previous point proves that $F$ is constant (and hence continuous) on $[0, q]$. Standard arguments (as in Burdett-Mortensen) prove continuity on $[\underline{q}, \bar{q}]$.

In the following sections we characterize the flow of transactions for any $F$ that satisfies Lemma 3 and then we characterize the seller's optimal quality choice $\hat{q}(c)$.

\subsubsection{Characterization of profits}

We take $H(\cdot), F(\cdot)$ and $\theta$ as given and calculate the steady state profits that a type- $c$ seller would enjoy for any quality $q$. The main result is summarized in the next proposition.

Proposition 4 The steady state profits of a seller of type c who offers quality $q$ are:

$$
\pi_{c}(q)= \begin{cases}\alpha_{B}(\theta) \theta p & \text { if } q<\underline{R} \\ \alpha_{B}(\theta) \theta\left(1+\frac{\phi \delta H(q)}{\left(\delta+\alpha_{B}(\theta)(1-F(q))\right)^{2}}\right)(p-c q) & \text { if } q \geq \underline{R} .\end{cases}
$$

To determine profits, we need to first determine the flow of a seller's transactions as a function of the quality he offers. The rate at which an individual seller is contacted by a new buyer is:

$$
t_{N}=\alpha_{S}(\theta)=\theta \alpha_{B}(\theta)
$$

The flow of transactions from loyal buyers is given by:

$$
t_{L}(q)=\phi l(q)
$$


where $l(q)$ is the steady steady number of loyal buyers of a seller offering $q$. Notice that unmatched buyers consume at rate $\alpha_{B}(\theta)$ and matched buyers consume at rate $\phi+\alpha_{B}(\theta)$ where $\phi$ is provided by their regular seller and $\alpha_{B}(\theta)$ is provided by new sellers.

The number of loyal buyers per seller offering $q$ is given by:

$$
l(q)=\frac{(B-\bar{n}) G^{\prime}(q)}{S F^{\prime}(q)}
$$

where $\bar{n}$ is the number of unmatched buyers, $(B-\bar{n}) G^{\prime}(q)$ is the number of buyers who are matched with a seller offering $q$ and $S F^{\prime}(q)$ is the number of sellers offering quality $q$.

We determine the number of unmatched buyers and their type distribution. In steady state, the flow of buyers from the unmatched to the matched state must equal the flow out of the matched state and into the unmatched state. Let $n(R)$ denote the number of buyers who are unmatched and whose type is less than $R$. The total number of unmatched buyers is therefore given by $n(\bar{R}) \equiv \bar{n}$.

An unmatched buyer of type $R$ becomes matched after transacting with a seller who offers above-reservation quality which occurs at rate $\alpha_{B}(\theta)(1-F(R))$. A matched buyer exits the matched state when his match is exogenously destroyed which occurs at rate $\delta$. As a result, in steady state the following holds:

$$
n^{\prime}(R) \alpha_{B}(\theta)(1-(F(R)))=\delta\left(B H^{\prime}(R)-n^{\prime}(R)\right) \Rightarrow n^{\prime}(R)=\frac{\delta B H^{\prime}(R)}{\delta+\alpha_{B}(\theta)(1-F(R))} .
$$

Hence, the mass $n(R)$ satisfies:

$$
n(R)=\int_{\underline{R}}^{R} \frac{B \delta}{\delta+\alpha_{B}(\theta)(1-F(x))} d H(x),
$$

and, thus, the mass of matched buyers is:

$$
\begin{aligned}
B-\bar{n} & =B\left(1-\int_{\underline{R}}^{\bar{R}} \frac{\delta}{\delta+\alpha_{B}(\theta)(1-F(x))} d H(x)\right) \\
& =\int_{\underline{R}}^{\bar{R}} \frac{B \alpha_{B}(\theta)(1-F(x))}{\delta+\alpha_{B}(\theta)(1-F(x))} d H(x) .
\end{aligned}
$$

We now characterize $G(\cdot)$. The mass of matched buyers receiving quality up to $q$ is given by $(B-\bar{n}) G(q)$. An unmatched type- $R$ buyer flows into this group if $R \leq q$ and he samples 
a seller who offers quality less than $q$, which occurs at rate $\alpha_{B}(\theta)(F(q)-F(R))$. A buyer flows out of this group if the match is exogenously destroyed or if he samples a new seller whose quality if greater than $q$, which occurs at rate $\delta+\alpha_{B}(\theta)(1-F(q))$. Equating these flows yields:

$$
\begin{aligned}
\alpha_{B}(\theta) \int_{\underline{R}}^{q}(F(q)-F(x)) d n(x) & =(B-\bar{n}) G(q)\left(\delta+\alpha_{B}(\theta)(1-F(q))\right) \\
\Rightarrow(B-\bar{n}) G(q) & =\frac{\alpha_{B}(\theta) \int_{\underline{R}}^{q}(F(q)-F(x)) d n(x)}{\delta+\alpha_{B}(\theta)(1-F(q))} \\
& =\frac{\alpha_{B}(\theta) B \delta \int_{\underline{R}}^{q} \frac{F(q)-F(x)}{\delta+\alpha_{B}(\theta)(1-F(x))} d H(x)}{\delta+\alpha_{B}(\theta)(1-F(q))} .
\end{aligned}
$$

Thus, $G^{\prime}(q)$ satisfies:

$$
(B-\bar{n}) G^{\prime}(q)=\frac{\alpha_{B}(\theta) B \delta F^{\prime}(q) H(q)}{\left(\delta+\alpha_{B}(\theta)(1-F(q))\right)^{2}},
$$

which implies that the flow of transactions from loyal buyers is:

$$
t_{L}(q)=\frac{\phi \alpha_{B}(\theta) \theta \delta H(q)}{\left(\delta+\alpha_{B}(\theta)(1-F(q))\right)^{2}} .
$$

Combining results completes the proof of Proposition 4.

\subsubsection{The sellers' optimal quality choice}

We now characterize the distribution of offered qualities, $F(\cdot)$ and the number of sellers who enter the market taking as given the number of buyers $B$ and the distribution of their reservation values $H(\cdot)$.

Lemma 5 Consider sellers 1 and 2 with $c_{1}>c_{2}$ and denote their actions by $q_{1}$ and $q_{2}$. Then:

1. $q_{2}>0 \Rightarrow q_{2}>q_{1}$.

2. $q_{2}=0 \Rightarrow q_{1}=0$. 
Proof. The proof is by contradiction. Suppose that $q_{2}>0$ and $q_{2} \leq q_{1}$. Recall that profits are given by $\pi_{c}(q)=(p-c q) t(q)$.

Seller 1 chose quality $q_{1}$ over $q_{2}$. Therefore:

$$
\left(p-c_{1} q_{1}\right) t\left(q_{1}\right) \geq\left(p-c_{1} q_{2}\right) t\left(q_{2}\right) \Rightarrow p\left(t\left(q_{1}\right)-t\left(q_{2}\right)\right) \geq c_{1}\left(t\left(q_{1}\right) q_{1}-t\left(q_{2}\right) q_{2}\right) .
$$

Seller 2 chose quality $q_{2}$ over $q_{1}$. Therefore:

$$
\left(p-c_{2} q_{2}\right) t\left(q_{2}\right) \geq\left(p-c_{2} q_{1}\right) t\left(q_{1}\right) \Rightarrow p\left(t\left(q_{1}\right)-t\left(q_{2}\right)\right) \leq c_{2}\left(t\left(q_{1}\right) q_{1}-t\left(q_{2}\right) q_{2}\right),
$$

which yields the desired contradiction. Supposing that $q_{2}=0, q_{1}>0$ and going through the same steps, proves the second point.

One corollary of Lemma 5 is that $F(\hat{q}(c))=1-D(c)$.

We now characterize the marginal seller $c^{*}$ and the lowest positive quality that is offered, $\underline{q}$ (we know from the previous Lemma that $\underline{q}$ is offered by the $c^{*}$-seller). Two conditions need to be satisfied: first, $\underline{q}$ must give higher profits to $c^{*}$ than any other positive quality level; second, $\underline{q}$ must give the same profits to $c^{*}$ as zero quality. The proposition summarizes the result.

Proposition 6 Given $H(\cdot)$ and $\theta$, there is a unique seller type $c^{*}$ such that:

1. Sellers with $c>c^{*}$ offer zero quality: $\hat{q}(c)=0$.

2. Sellers with $c \leq c^{*}$ offer positive quality and the marginal seller $c^{*}$ offers the lowest positive quality $\underline{q}$.

3. The marginal seller is determined by the solution to:

$$
p=\left(p-c^{*} \underline{q}\left(c^{*}\right)\right)\left(1+\frac{\phi \delta H\left(\underline{q}\left(c^{*}\right)\right)}{\left(\delta+\alpha_{B}(\theta) D\left(c^{*}\right)\right)^{2}}\right),
$$

where $\underline{q}$ is the solution to:

$$
-c\left(1+\frac{\phi \delta H(\underline{q})}{\left(\delta+\alpha_{B}(\theta) D(c)\right)^{2}}\right)+(p-c \underline{q}) \frac{\phi \delta H^{\prime}(\underline{q})}{\left(\delta+\alpha_{B}(\theta) D(c)\right)^{2}}=0 .
$$


Denote the profits of a type- $c$ seller who offers the lowest positive quality level $\underline{q}$ by:

$$
\underline{\pi}_{c}(\underline{q})=\alpha_{B}(\theta) \theta(p-c \underline{q})\left(1+\frac{\phi \delta H(\underline{q})}{\left(\delta+\alpha_{B}(\theta) D(c)\right)^{2}}\right)
$$

for $\underline{q} \in[\underline{R}, \bar{R}]$. Since quality is decreasing in a seller's cost type and, by assumption, the type- $c$ seller offers the lowest positive quality level, we have $1-F(0)=D(c)$. Notice that the level of profits for this seller do not depend on the exact shape of $F(\cdot)$ over and above the mass at zero.

Denote the optimal choice of a type- $c$ seller who offers the lowest positive quality by $\underline{q}(c)$. This is determined as the root of

$$
\underline{\pi}_{c}^{\prime}(\underline{q})=\alpha_{B}(\theta) \theta\left[-c\left(1+\frac{\phi \delta H(\underline{q})}{\left(\delta+\alpha_{B}(\theta) D(c)\right)^{2}}\right)+(p-c \underline{q}) \frac{\phi \delta H^{\prime}(\underline{q})}{\left(\delta+\alpha_{B}(\theta) D(c)\right)^{2}}\right]
$$

assuming that the second order conditions hold:

$$
\underline{\pi}_{c}^{\prime \prime}(\underline{q})=\alpha_{B}(\theta) \theta \frac{-2 c \phi \delta H^{\prime}(\underline{q})+(p-c \underline{q}) \phi \delta H^{\prime \prime}(\underline{q})}{\left(\delta+\alpha_{B}(\theta) D(c)\right)^{2}}<0
$$

Suppose there exists a marginal seller $c^{*}$ who is indifferent between offering zero or the (optimally chosen) lowest positive quality:

$$
\pi_{c^{*}}(0)=\underline{\pi}_{c^{*}}\left(\underline{q}\left(c^{*}\right)\right)
$$

We show that offering $q \in\left(0, \underline{q}\left(c^{*}\right)\right)$ is suboptimal for all other sellers. Lemma 5 shows that offering $q<\underline{q}\left(c^{*}\right)$ is inconsistent with optimal behavior for a seller with $c<c^{*}$. Consider a seller with $c^{\prime *}$. Differentiating profits at the optimally chosen lowest positive quality with respect to $c$ we have:

$$
\frac{\partial \underline{\pi}_{c}(\underline{q}(c))}{\partial c}=\underline{\pi}_{c}^{\prime}(\underline{q}(c)) \frac{d \underline{q}(c)}{d c}-\underline{q}(c) t(q)<0
$$

The first term is zero by the envelope condition and the second term is negative because higher costs reduce margins.

As a result, if the $c^{*}$ seller is indifferent between 0 and $\underline{q}\left(c^{*}\right)$ we have:

$$
\underline{\pi}_{c^{\prime}}\left(\underline{q}\left(c^{\prime}\right)\right)<\underline{\pi}\left(\underline{q}\left(c^{*}\right)\right)=\pi_{c^{*}}(0)=\pi_{c^{\prime}}(0)
$$


and it is optimal for a seller with $c^{\prime *}$ to offer zero quality. This proves parts 2 and 3 of Proposition 6 .

We now show that $c^{*}$ exists and it is unique. Using our assumptions on the support of $D(\cdot)$ :

$$
\begin{array}{r}
\lim _{c \rightarrow \infty} \underline{\pi}_{c}(\underline{q}(c))=\lim _{c \rightarrow \infty}(p-c \underline{q}(c)) t(\underline{q}(c))<\lim _{c \rightarrow \infty} \pi_{c}(0) \\
\lim _{c \rightarrow 0} \underline{\pi}_{c}(\underline{q}(c))=\lim _{c \rightarrow 0} p t(\underline{q}(c))>\lim _{c \rightarrow \infty} \pi_{c}(0)
\end{array}
$$

As the type of the marginal seller changes, his profits change as follows:

$$
\frac{d \underline{\pi}_{c}(\underline{q}(c))}{d c}=\underline{\pi}_{c}^{\prime}(\underline{q}(c)) \frac{d \underline{q}(c)}{d c}+\frac{\partial \underline{\pi}_{c}(\underline{q}(c))}{\partial c}+\frac{\partial \underline{\pi}_{c}(\underline{q}(c))}{\partial D(c)} D^{\prime}(c)<0 .
$$

The first and second terms are negative for the same reasons as above. The third term is negative because

$$
\frac{\partial \underline{\pi}_{c}(\underline{q}(c))}{\partial D(c)}=\alpha_{B}(\theta) \theta \frac{(p-c \underline{q}(c)) \phi \delta H(\underline{q}(c)) 2 \alpha_{B}(\theta)}{\left(\delta+\alpha_{B}(\theta) D(c)\right)^{4}}>0
$$

Thus, there is a unique $c^{*}$ such that the profits from offering $\underline{q}\left(c^{*}\right)$ are exactly equal to the profits from offering zero.

Finally, equating $\pi_{c^{*}}(0)$ with $\underline{\pi}_{c^{*}}\left(\underline{q}\left(c^{*}\right)\right)$ and going through the algebra yields:

$$
p=\left(p-c^{*} \underline{q}\left(c^{*}\right)\right)\left(1+\frac{\phi \delta H\left(\underline{q}\left(c^{*}\right)\right)}{\left(\delta+\alpha_{B}(\theta) D\left(c^{*}\right)\right)^{2}}\right)
$$

where $\underline{q}\left(c^{*}\right)$ is defined by the root of equation (6).

Therefore, the optimal quality choice for sellers with $c>c^{*}$ is $\hat{q}=0$ and for $c=c^{*}$ it is $\hat{q}=\underline{q}\left(c^{*}\right)$. This completes the proof of Proposition 6 .

We now determine $\hat{q}(c)$ for $c<c^{*}$.

Proposition 7 Given $H(\cdot)$ and $\theta$, the optimal quality choice for sellers of type $c<c^{*}$ solves the differential equation:

$$
\hat{q}^{\prime}(c)=-\frac{2 \phi \delta\left(\frac{p}{c}-\hat{q}(c)\right) H(\hat{q}(c)) \alpha_{B}(\theta) D^{\prime}(c)}{\left(\delta+\alpha_{B}(\theta) D(c)\right)\left(\left(\delta+\alpha_{B}(\theta) D(c)\right)^{2}+\phi \delta H(\hat{q}(c))-\phi \delta\left(\frac{p}{c}-\hat{q}(c)\right) H^{\prime}(\hat{q}(c))\right)}
$$


with initial condition $\underline{q}\left(c^{*}\right)$. The distribution of qualities is given by:

$$
F(q)=1-D\left(\hat{q}^{-1}(q)\right) .
$$

To characterize the function of optimal quality offer $\hat{q}(c)$ we rewrite the profits of a type- $c$ seller as if he decides which other type $c^{\prime}$ to imitate rather than which quality to offer. In other words, his profits from offering some quality $q^{\prime}$ are written in terms of imitating type $c^{\prime}$ who offers quality $q^{\prime}=\hat{q}\left(c^{\prime}\right)$. We have:

$$
\pi_{c}\left(c^{\prime}\right)=\alpha_{B}(\theta) \theta\left(p-c \hat{q}\left(c^{\prime}\right)\right)\left(1+\frac{\phi \delta H\left(\hat{q}\left(c^{\prime}\right)\right)}{\left(\delta+\alpha_{B}(\theta) D\left(c^{\prime}\right)\right)^{2}}\right)
$$

The advantage of formulating the choice in terms of $c^{\prime}$ rather than $q^{\prime}$ is that the term in the denominator depends on the exogenous type distribution $D(\cdot)$ rather than the endogenous quality distribution $F(\cdot)$. The quality distribution will be recovered once $\hat{q}(c)$ is constructed.

Differentiate profits with respect to $c^{\prime}$

$$
\begin{aligned}
\pi_{c}^{\prime}(\hat{c} ; c)= & \alpha_{B}(\theta) \theta c\left(-\hat{q}^{\prime}\left(c^{\prime}\right)\left(1+\frac{\phi \delta H\left(\hat{q}\left(c^{\prime}\right)\right)}{\left(\delta+\alpha_{B}(\theta) D\left(c^{\prime 2}\right.\right.}\right)\right. \\
& \left.+\left(\frac{p}{c}-\hat{q}\left(c^{\prime}\right)\right) \phi \delta \frac{H^{\prime}\left(\hat{q}\left(c^{\prime}\right)\right) \hat{q}^{\prime}\left(c^{\prime}\right)\left(\delta+\alpha_{B}(\theta) D\left(c^{\prime}\right)\right)-H\left(\hat{q}\left(c^{\prime}\right)\right) 2 \alpha_{B}(\theta) D^{\prime}\left(c^{\prime}\right)}{\left(\delta+\alpha_{B}(\theta) D\left(c^{\prime}\right)\right)^{3}}\right)
\end{aligned}
$$

By construction, profits are maximized when $\hat{c}=c$ and we can therefore set the derivative to zero and rearrange to arrive at equation (7). This differential equation determines $\hat{q}(c)$. This completes the proof.

Having fully characterized $F(\cdot)$, we turn to determining the number of sellers $S$ who choose to enter the market.

Proposition 8 Given $H(\cdot)$ and $B$ there is a unique $S$ such that $\Pi=K_{S}$.

The key for this proposition is that profits for every type of seller are increasing in $\theta$ :

$$
\frac{d \pi_{c}(q)}{d \theta}=\frac{\partial \pi_{c}(q)}{\partial \theta}+\frac{\partial \pi_{c}(q)}{\partial q} \frac{d q}{d \theta} .
$$


The first term is clearly positive. The second terms is zero by the envelope theorem. Furthermore:

$$
\begin{aligned}
& \lim _{\theta \rightarrow 0} \pi_{c}(q)=0, \\
& \lim _{\theta \rightarrow \infty} \pi_{c}(q)>K_{S},
\end{aligned}
$$

which proves Proposition 8 .

This completes the characterization of sellers' behavior.

\section{Quantitative Analysis}

The model does not admit an analytic solution for all endogenous outcomes. Hence, we choose the parameters that best match moments of the data with the corresponding moments computed from the model's numerical solution. We then study the quantitative implications of the model evaluated at the estimated parameters.

\subsection{Estimation and Identification}

We estimate the model using the data described in Section 2, assuming that they are generated from the model's steady state. We set the unit of time to be one month.

Unfortunately, the data lack some detailed information to identify all parameters. Therefore, we fix some values. Specifically, the discount rate $r$ is traditionally difficult to identify, and we set it to $r=.01$. Moreover, since we use the normalized the variable Pure Grams PER $\$ 100$, we set the price to be equal to $p=\$ 100$. Furthermore, we set sellers' monthly opportunity cost $K_{S}$ to be $\$ 2,000$, which is broadly in line with drug-dealers' average earnings reported by Levitt and Venkatesh (2000).

We further make parametric assumptions about the distributions of buyers' and of sellers' heterogeneity. ${ }^{8}$ We assume that the distribution $M(\cdot)$ of buyers' taste for drugs $z$ is lognormal with unknown parameters $\mu_{z}$ and $\sigma_{z}$. This implies that the distribution $H(\cdot)$ of reservation qualities $R=\frac{p}{z}$ is also lognormal with parameters $\mu_{R}=\log p-\mu_{z}$ and $\sigma_{z}$. Moreover, we assume that the distribution of the inverse of sellers' costs $1 / c$ follow a Pareto

\footnotetext{
${ }^{8}$ If quality $q(c)$ is a strictly monotonic (and, thus, invertible) function of cost $c$, we can estimate directly the distribution $D(c)$ from the empirical distribution of $q$ : $D(c)=1-F(q)$. However, we specify a parametric distribution for $D(c)$ because several sellers with different costs $c$ choose $q(c)=0$.
} 
distribution with lower bound $\frac{1}{c_{M}}$ and shape parameter $\xi \geq 1$. This implies that the distribution of $\operatorname{costs} c$ is:

$$
D(c)=\left(\frac{c}{c_{M}}\right)^{\xi}, c \in\left[0, c_{M}\right] .
$$

The shape parameter $\xi$ captures the dispersion of costs. If $\xi=1$, the cost distribution is uniform on $\left[0, c_{M}\right]$. As $\xi$ increases, the relative number of high-cost sellers increases, and the cost distribution is more concentrated at these higher cost levels. As $\xi$ goes to infinity, the distribution becomes degenerate at $c_{M}$.

Finally, we assume that drug qualities $q$ are measured with error. More specifically, we assume that the reported qualities $q^{*}$ and the "true" qualities $q$ are related as:

$$
q^{*}=q \epsilon
$$

where $\epsilon$ is a measurement error. We assume that $\epsilon$ has a lognormal distribution and restrict its mean to be equal equal to 1 , which implies that the parameters $\mu_{\epsilon}$ and $\sigma_{\epsilon}$ of the lognormal distribution satisfy $\mu_{\epsilon}=-.5 \sigma_{\epsilon}^{2}$. The assumption of measurement error on wages is quite common in the literature that structurally estimates search models of the labor market. Here, it allows us to fit better the quality distribution. In particular, as figures 4 and 5 display, the model implies a gap in the quality distribution between the complete rip-offs $q=0$ and the minimum positive quality $\underline{q}$. While figure 2 shows that the empirical distribution displays this qualitative feature, the measurement $\epsilon$ allows it to more precisely match its magnitude.

We estimate the vector of parameters $\psi=\left\{\alpha, \phi, \delta, K_{B}, \mu_{R}, \sigma_{R}, c_{M}, \xi, \sigma_{\epsilon}\right\}$ using a minimumdistance estimator that matches key moments of the data with the corresponding moments of the model. More precisely, for any value of these parameters, we solve the model of Section 3 to find its equilibrium: the mass $B$ of active buyers and their distribution of reservation qualities $H(\cdot)$, and the mass $S$ of active sellers and their distribution $F(\cdot)$ of offered qualities. We further simulate the model to calculate buyers' distributions of consumptions in one period. We then calculate the vector $m(\psi)$ composed by these moments:

1. The fraction of rip-offs: ${ }^{9}$

$$
m_{1}=F(q=0)
$$

\footnotetext{
${ }^{9}$ Note that $q^{*}=0$ if and only if $q=0$. Thus, the fraction of rip-offs is equal to $F(q=0)$.
} 
2. The mean of quality for $q^{*}>0$ :

$$
m_{2}=E\left(q^{*} \mid q^{*}>0\right)
$$

3. The standard deviation of quality for $q^{*}>0$ :

$$
m_{3}=\sqrt{\operatorname{Var}\left(q^{*} \mid q^{*}>0\right)} .
$$

4. The median of quality for $q^{*}>0$ :

$$
m_{4}=\left(q_{m}^{*}: \operatorname{Pr}\left(q^{*} \leq q_{m}^{*} \mid q^{*}>0\right)=\frac{1}{2}\right) .
$$

5. The skewness of quality for $q^{*}>0$ :

$$
m_{5}=E\left[\left(\frac{q^{*}-E\left(q^{*} \mid q^{*}>0\right)}{\sqrt{\operatorname{Var}\left(q^{*} \mid q^{*}>0\right)}}\right)^{3} \mid q^{*}>0\right]
$$

6. The kurtosis of quality for $q^{*}>0$ :

$$
m_{6}=E\left[\frac{\left(q^{*}-E\left(q^{*} \mid q^{*}>0\right)\right)^{4}}{\left(\operatorname{Var}\left(q^{*} \mid q^{*}>0\right)\right)^{2}} \mid q^{*}>0\right] .
$$

7. The fraction of matched buyers:

$$
m_{7}=1-\frac{\bar{n}}{B}=\int_{\underline{R}}^{\bar{R}} \frac{\alpha(1-F(R))}{\delta+\alpha(1-F(R))} d H(R) .
$$

8. The average number of purchases of those who are matched to a regular dealer:

$$
m_{8}=E(\text { contacts } \mid \text { matched }) \text {. }
$$

9. The average number of purchases of those who are not matched a regular dealer:

$$
m_{9}=E(\text { contacts } \mid \text { unmatched }) .
$$


10. The standard deviation of the number of purchases of those who are matched to a regular dealer:

$$
m_{10}=\sqrt{\text { Var (contacts } \mid \text { matched })}
$$

11. The standard deviation of the number of purchases of those who are not matched a regular dealer:

$$
m_{11}=\sqrt{\operatorname{Var}(\text { contacts } \mid \text { unmatched })} .
$$

The minimum-distance estimator chooses the parameter vector $\psi$ that minimizes the criterion function

$$
\left(m(\psi)-m_{S}\right)^{\prime} \Omega\left(m(\psi)-m_{S}\right),
$$

where $m(\psi)$ is the vector of moments computed from the model evaluated at $\psi$, and $m_{S}$ is the vector of corresponding sample moments. $\Omega$ is a symmetric, positive-definite weighting matrix. In practice, we use the identity matrix.

Although the model is highly nonlinear, so that (almost) all parameters affect all outcomes, the identification of some parameters relies on some key moments in the data. Specifically, the moments of the quality distribution identify the parameters of the distribution $D$ of sellers' heterogeneity, of the distribution of the measurement error, and contribute to the identification of the parameters of the distribution $H$ of buyers' heterogeneity. The moments of buyers' consumptions identify the meeting rates $\alpha$ and $\phi$, the destruction rate $\delta$, and contribute to the identification of the parameters of the distribution $H$ of buyers' heterogeneity. From the distribution of buyers' heterogeneity, we can then recover buyers' cost $K_{B}$. Finally, given the estimated parameters, we can further recover the buyers-sellers ratio $\theta$ from sellers' free-entry condition.

\subsubsection{Estimates}

Table 2 reports estimates of the parameters, along with 95-percent confidence intervals obtained by bootstrapping the data using 100 replications (to be computed).

The magnitude of the parameter $\alpha$ indicates that a buyer meets a new seller, on average, every $\frac{30}{\alpha}=25$ days. The parameter $\phi$ indicates that a matched buyer purchases, on average, approximately 10 times every month. However, the buyer-seller match lasts, on average, only $\frac{30}{\delta}=40$ days. Moreover, the parameter $\theta$ indicates that a seller serves, on average, approximately 9 buyers. Buyers' monthly cost $K_{B}$ is quite low, approximately equal to $\$ 55$. 
TABle 2: Parameter Estimates

\begin{tabular}{cccc}
\hline$\alpha$ & 1.1554 & 3.6919 \\
& {$[]$,} & $\mu_{z}$ & {$[]$,} \\
$\phi$ & 9.3874 & & 0.1122 \\
& {$[]$,} & $\sigma_{z}$ & {$[]$,} \\
$\delta$ & 0.7356 & & 22.8502 \\
& {[]} & $c_{M}$ & {$[]$,} \\
& 0.2481 & & 4.5762 \\
$\sigma_{\epsilon}$ & {[]} & $\xi$ & {$[]$,} \\
& 54.2760 & & 9.0566 \\
$K_{B}$ & {$[]$,} & $\theta$ & {$[]$,} \\
\hline \hline
\end{tabular}

Notes - This table reports the estimates of the parameters. 95-percent confidence intervals in brackets are obtained by bootstrapping the data using 100 replications (to be computed).

The parameters $c_{M}$ and $\xi$ of sellers' cost distribution imply that the range of sellers' cost is $[0,22.8502]$, but their average cost is 18.75 , as $\xi=4.5762$ implies that most sellers have costs close to the upper bound $c_{M}$. Moreover, the estimates of the parameters of the distribution of buyers' heterogeneity imply that all buyers with taste $z \geq z^{*}=25.4179$ are active in the market and, among those active, the average taste is approximately equal to 40 and the standard deviation is approximately equal to 10.

Finally, the variance of the measurement error is estimated to be quite small, indicating that the model without any error already captures the data quite well.

\subsubsection{Model Fit}

Before considering some broader implications of our results, we examine the fit of the estimated model. Table 3 presents a comparison between the empirical moments and the moments calculated from the model at preliminary parameters. Overall, the model matches the moments of the quality distribution quite well. The largest discrepancy is in the variance of the offered qualities, that is the model implies a dispersion of the offered qualities that is lower than that of the observed distribution. Nonetheless, the model captures well both the fraction of ripoffs and the higher-order moments of the quality distribution. The model matches the moments of the distribution of buyers' consumptions less precisely than those of the quality distribution, but overall it captures quite well the difference in consumption rates between matched and unmatched buyers. 
TABle 3: Model Fit

\begin{tabular}{lcc}
\hline \hline & Data & Model \\
\hline Fraction of Rip-offs & 0.0608 & 0.0657 \\
Average Pure Grams Per $\$ 100, q^{*}>0$ & 3.3374 & 3.9775 \\
St. Dev. Pure Grams Per $\$ 100, q^{*}>0$ & 2.0548 & 1.2107 \\
Median Pure Grams Per $\$ 100, q^{*}>0$ & 2.9502 & 3.8028 \\
Skewness Pure Grams Per $\$ 100, q^{*}>0$ & 1.0183 & 0.8720 \\
Kurtosis Pure Grams Per $\$ 100, q^{*}>0$ & 3.7428 & 4.0935 \\
Fraction Of Matched Buyers & 0.5526 & 0.6012 \\
Average Number of Purchases, Matched Buyer & 6.3774 & 8.7742 \\
Average Number of Purchases, Unmatched Buyer & 4.3309 & 6.3542 \\
St. Dev. Number of Purchases, Matched Buyer & 7.1249 & 3.9915 \\
St. Dev. Number of Purchases, Unmatched Buyer & 5.4303 & 3.5400 \\
\hline \hline
\end{tabular}

Notes - This table reports the values of the empirical moments and of the simulated moments calculated at the estimated parameters reported in Table 2.

To further appreciate how the model compares to the quality data in a perhaps moreintuitive way, Figure 3 displays the histogram of the quality distribution obtained from a model simulation using the estimated parameters reported in Table 2. The comparison with the empirical distribution of Figure 2 corroborates that the model matches the qualitative and quantitative features of the distribution of drug qualitiy quite well.

\subsection{Model Implications}

Figure 4 displays sellers' choice of quality as a function of their costs $c$. For sellers with costs $c \in\left[0, c^{*}\right], q(c)$ is the solution to the differential equation (7): sellers' quality choices are strictly decreasing in their costs, as Lemma 5 says. Instead, all sellers with costs $c \in\left(c^{*}, c_{M}\right]$ choose to rip-off their buyers by choosing $q=0$. While the interval $\left(c^{*}, c_{M}\right]$ is small in the figure, the mass of sellers in that interval is relatively larger, because the shape parameter $\xi$ of the Pareto distribution is quite large. Sellers' quality choice $q(c)$ implies that sellers' markups $\frac{p-c q(c)}{p}$ are non-monotonic, with the lowest- and highest-cost sellers charging the highest ones (equal to 1 , as either $c$ or $q$ equals 0 ) and a seller with cost $c=17.39$ charging the lowest one; the average sellers' markup $\frac{\int_{0}^{c} M(p-c q(c)) d D(c)}{p}$ equals approximately 35 percent. 


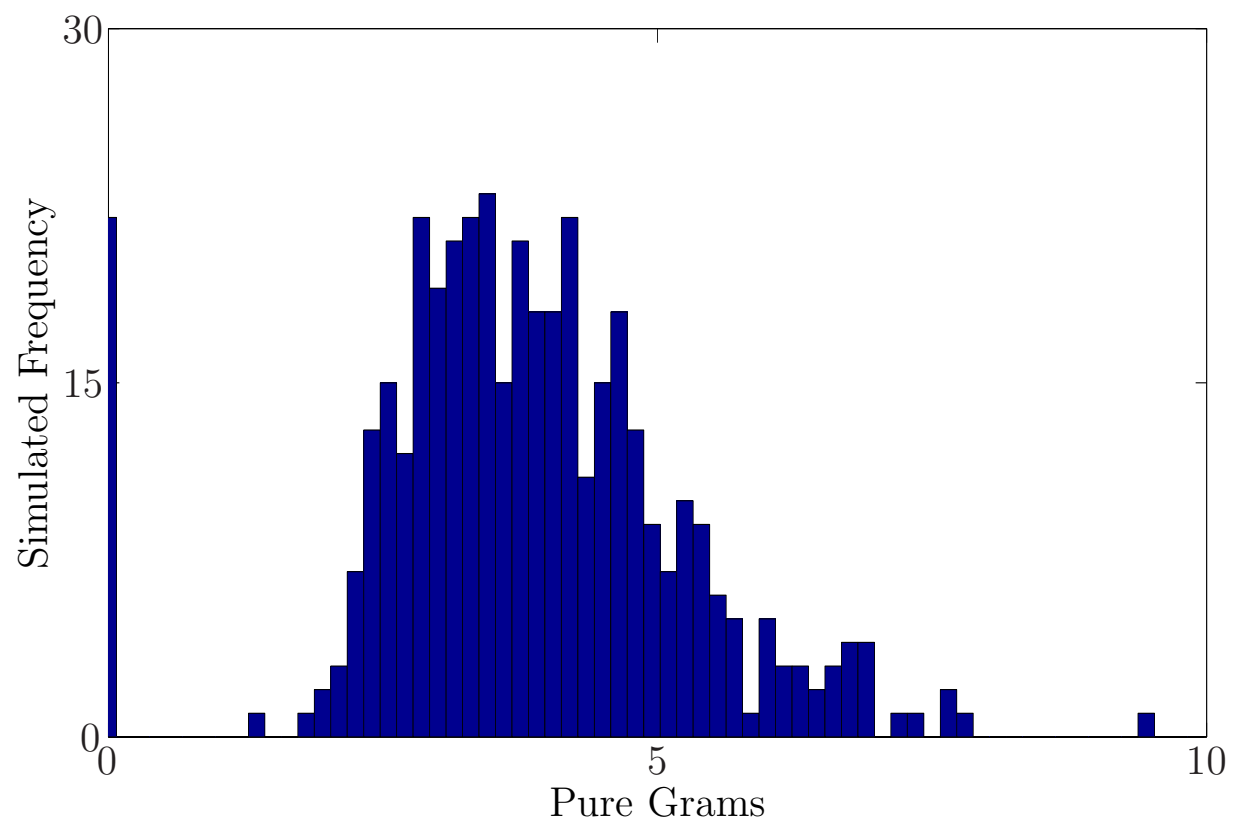

FIG. 3: Histogram of pure grams per $\$ 100$, simulated data.

On average, sellers make approximately 65 transactions $t(q)$ per month, and the distribution of transactions $t(q)$ has a large range - the lowest-quality (i.e., higher-cost) sellers make approximately 10 monthly deals and the highest-quality sellers make approximately 150 monthly deals - and is skewed towards sellers with fewer transactions. Sellers' profits have a large range and are highly skewed as well: the lowest-quality' seller is earning approximately $\$ 1,000$ per month, the highest-quality' seller is earning approximately $\$ 15,000$ per month, and the average seller is earning $K_{S}=\$ 2,000$. The shape of the distribution of profits matches reasonably well the descriptive evidence reported by Levitt and Venkatesh (2000).

Figure 5 compares the equilibrium distribution of qualities consumed by first-time (i.e., unmatched) buyers and the equilibrium distribution of qualities consumed by regular (i.e., matched) buyers. The left panel displays the key features of the distribution of qualities $F(q)$ characterized in Lemma 3, most notably the mass point at $q=0$. Of course, no matched buyers consumes $q=0$ from his regular dealer. Moreover, as buyers move up over time in the offered quality distribution by switching to sellers that offer higher-quality drugs, they are more likely to be matched to higher-quality sellers. Hence, the cumulative $G(q)$ first-order stochastically dominates the cumulative $F(q)$. The right panel compares the probability density functions $f(q)$ and $g(q)$ on $[\underline{q}, \bar{q}]$, indicating that, at the estimated parameters, buyers' switching behavior has a large effect on the distribution of qualities that regular buyers are consuming relative to the distribution of qualities that first-time buyers 


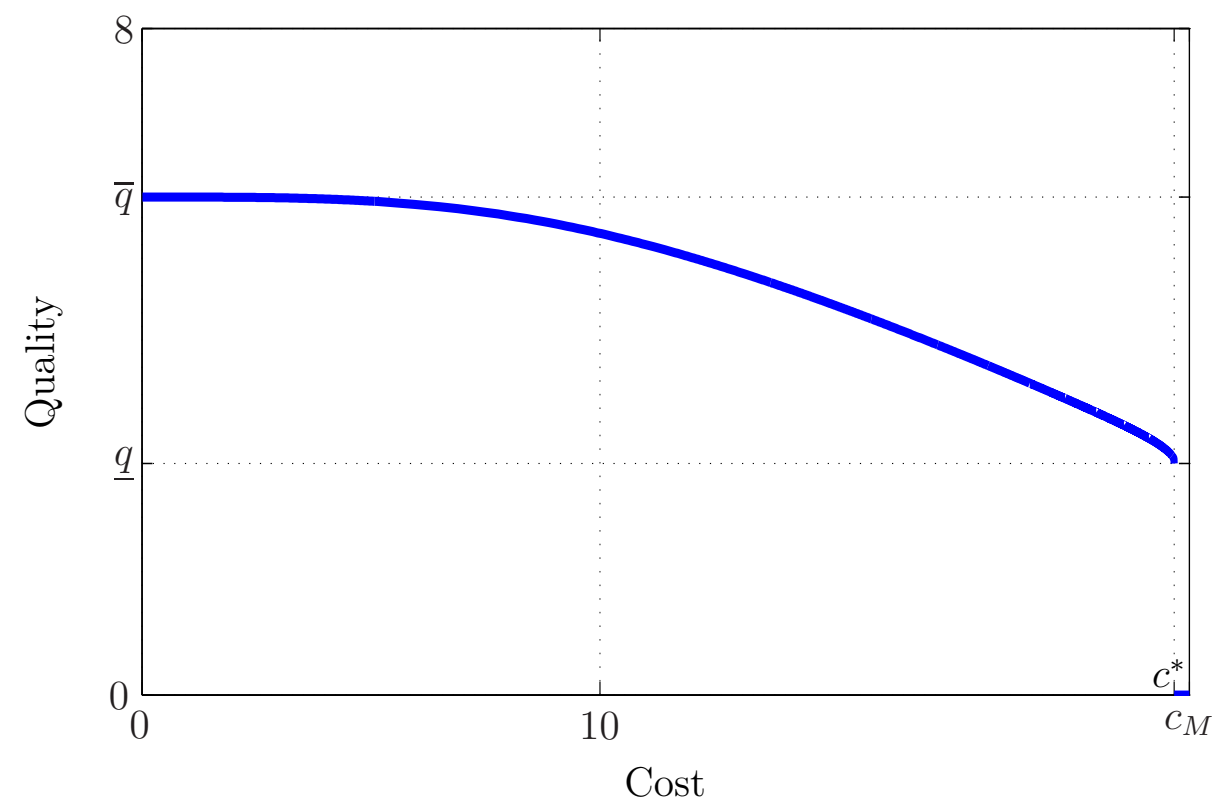

FIG. 4: Sellers' quality choice as a function of their cost. Based on the parameter estimates reported in Table 2 .

are consuming.

\subsubsection{The Role of Sellers' Moral Hazard}

In this Section, we use our model to understand how sellers' moral hazard due to the imperfect observability of drugs' purity affects market outcomes. To do so, we modify the model of Section to allow buyers to observe drug purity before purchasing it. Appendix A reports the full derivation of the equilibrium. We highlight here how the observability of $q$ modifies buyers' values, sellers' profits and, thus, the equilibrium distribution of quality $q$.

When buyers observe $q$ before transacting, they purchase only if the seller offers a sufficiently high $q$, so that their flow payoff $z q-p$ is non-negative. Thus, their value functions are:

$$
\begin{aligned}
r \bar{V}_{z} & =\alpha_{B}(\theta) \int_{0}^{\bar{q}}\left(\max \left[z \tilde{q}-p+\max \left[V_{z}(\tilde{q})-\bar{V}_{z}, 0\right], 0\right]\right) d F(\tilde{q}), \\
r V_{z}(q) & =\phi(z q-p)+\alpha_{B}(\theta) \int_{0}^{\bar{q}}\left(\max \left[z \tilde{q}-p+\max \left[V_{z}(\tilde{q})-V_{z}(q), 0\right], 0\right]\right) d F(\tilde{q})+\delta\left(\bar{V}_{z}-V_{z}(q)\right)
\end{aligned}
$$

Buyers' purchase decisions affect sellers profits, as well. Specifically, the rate at which an individual seller offering quality $q$ transacts with a new buyer depends on the meeting rate $\alpha_{S}(\theta)$ and on the probability $H(q)$ that the seller's quality $q$ is above the buyer's reservation 

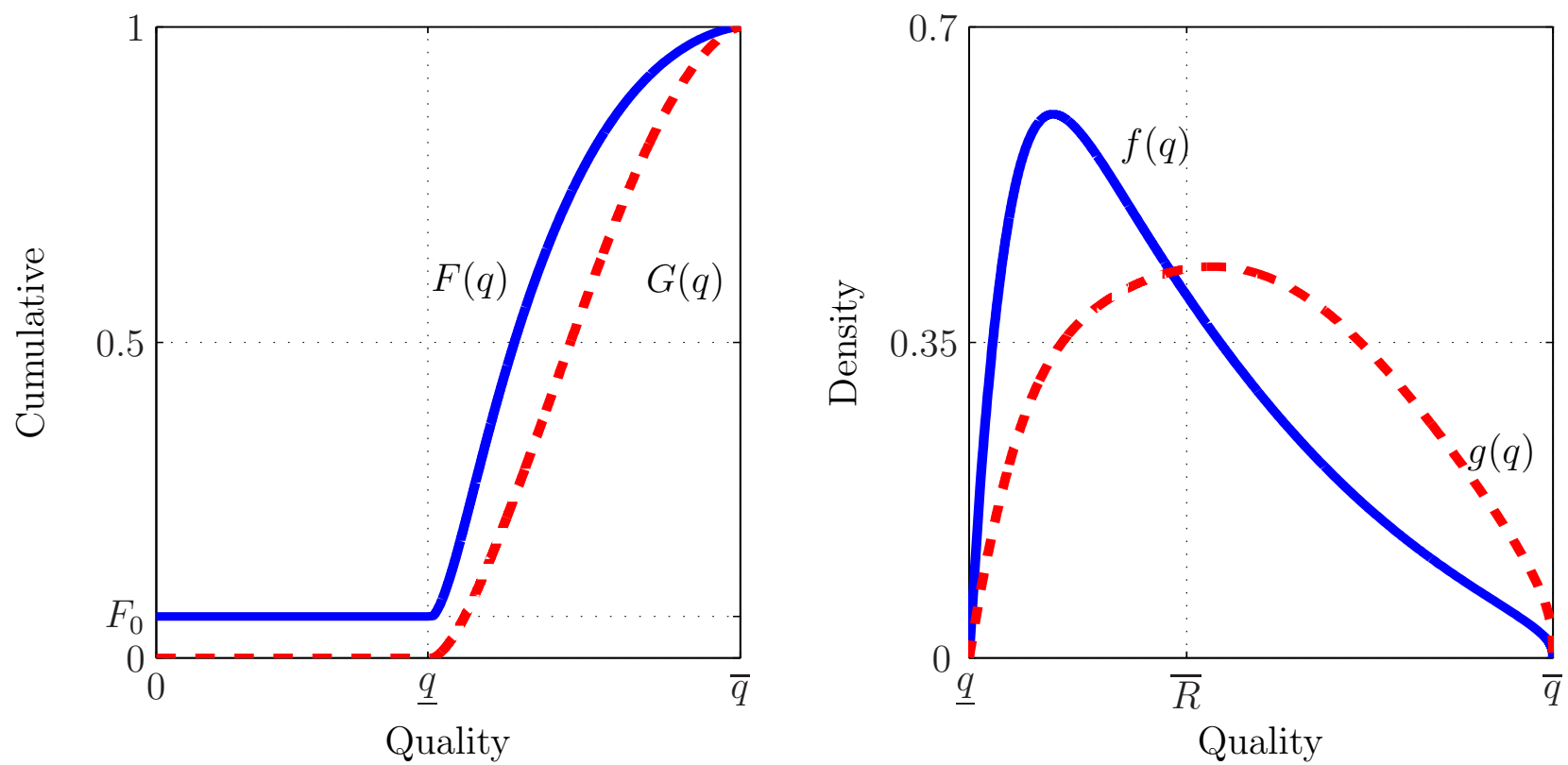

FIG. 5: The left panel displays the cumulative distribution functions of quality $F(q)$ (solid line) and $G(q)$ (dashed line). The right panel displays the probability density functions of quality $f(q)$ (solid line) and $g(q)$ (dashed line) on $[\underline{q}, \bar{q}]$. Based on the parameter estimates reported in Table 2.

value:

$$
t_{N}(q)=\alpha_{S}(\theta) H(q)=\alpha_{B}(\theta) \theta H(q) .
$$

Since loyal buyers know the quality that the seller is offering, equation (5) still characterizes the rate $t_{L}(q)$ at which an individual seller offering quality $q$ transacts with loyal buyers. Therefore, sellers' steady state profits are:

$$
\pi_{c}(q)=\alpha_{B}(\theta) \theta H(q)(p-c q)\left(1+\frac{\phi \delta}{\left(\delta+\alpha_{B}(\theta)(1-F(q))\right)^{2}}\right), \quad q \geq \underline{q} .
$$

Hence, sellers' optimal quality choice is determined by the following differential equation

$$
\hat{q}^{\prime}(c)=\frac{2(p-c \hat{q}(c)) \phi \delta \alpha_{B}(\theta) D^{\prime}(c)}{\left[\frac{H^{\prime}(\hat{q}(c))}{H(\hat{q}(c))}(p-c \hat{q}(c))-c\right]\left(\delta+\alpha_{B}(\theta) D(c)\right)\left[\left(\delta+\alpha_{B}(\theta) D(c)\right)^{2}+\phi \delta\right]}
$$

with initial condition $\underline{q}\left(c^{*}\right)$, determined by the profit-maximazation of the highest-cost seller $c^{*}:$

$$
\max _{q} \pi\left(c^{*}\right)=\max \alpha_{B}(\theta) \theta H(\underline{q})\left(p-c^{*} \underline{q}\right)\left(1+\frac{\phi \delta}{\left.\left(\delta+\alpha_{B}(\theta)\right)\right)^{2}}\right) .
$$


TABLE 4: Counterfactuals: Observable Quality

\begin{tabular}{|c|c|c|c|}
\hline & BASELINE & $\begin{array}{l}\text { OBSERVABle } q \text {, } \\
\text { PARTIAL Eq. }\end{array}$ & $\begin{array}{l}\text { OBSERVABLE } q \text {, } \\
\text { GENERAL EQ. }\end{array}$ \\
\hline FRACTION OF RIP-OFFS & 0.0657 & 0 & 0 \\
\hline Average Pure Grams per $\$ 100$ & 3.7463 & 4.0388 & 4.0277 \\
\hline VARIANCE PURE GRAMs PER $\$ 100$ & 1.3242 & 0.6760 & 0.6542 \\
\hline Mass of Active Buyers & $4.0022 * 10^{6}$ & $4.0022 * 10^{6}$ & $4.0022 * 10^{6}$ \\
\hline Mass of ACtive SEllers & $4.4191 * 10^{5}$ & $4.4191 * 10^{5}$ & $4.1015 * 10^{5}$ \\
\hline Fraction of Matched Buyers & 0.6012 & 0.6198 & 0.6078 \\
\hline Average Number of Purchases & 6.9650 & 7.2600 & 7.0950 \\
\hline Average Pure Grams Consumed & 28.6241 & 30.2059 & 29.4732 \\
\hline
\end{tabular}

Notes-This table reports market outcomes in the counterfactual cases in which buyers can observe drugs' purity before purchasing.

Thus, the equilibrium distribution of qualities is:

$$
F(q)=1-D\left(\hat{q}^{-1}(q)\right) .
$$

We compute the resulting equilibria for two alternative cases: 1) a partial-equilibrium case in which buyers make optimal purchase decisions and sellers choose the optimal quality to offer, but buyers' and sellers' masses and types are unchanged relative to the benchmark case; and 2) a general-equilibrium case in which, in addition to the partial-equilibrium optimizations, buyers and sellers also make optimal entry decisions - i.e, a buyer's entry threshold $z^{* *}$ satisfy $r \bar{V}_{z}=K_{B}$ the and the mass of sellers $S^{* *}$ satisfies $\int \pi(q(c)) d D(c)=K_{S}$. We believe that the partial-equilibrium case is useful to focus exclusively on the effects of sellers' moral hazard due the imperfect observability of drugs' purity.

Table 4 reports the quantitative values of market outcomes for the counterfactuals of observable drug purity for the partial-equilibrium case and the general-equilibrium case. Overall, the quantitative effects are very similar in these two cases. However, market outcomes differ substantially when buyers observe drug purity and when they do not. Specifically, if drug purity is observable, sellers cannot rip-off buyers and, thus, zero-purity drugs disap- 
pear from the market. Moreover, sellers have to increase quality to induce their first-time occasional buyers to purchase and, possibly, to become their loyal customers and, thus, the average purity increases by approximately eight percent and the variance of purity decreases by almost 50 percent. Hence, a larger fraction of buyers is matched to a regular seller, thereby increasing buyers' purchases and consumption by approximately five percent.

The general equilibrium case highlights three additional effects relative to the partialequilibrium case. When buyers can observe drug quality, their average purity increases, thereby attracting a larger number of active buyers relative to the baseline case. However,

since it is more expensive to supply higher-quality drugs, sellers' profits decrease relative to the baseline case. Hence, in equilibrium, fewer sellers enter the market. In turn, it becomes more difficult for buyers to meet new sellers, thereby decreasing their purchases and, thus, affecting their consumption, as well. Overall, the quantitative differences between the baseline model with unobservable quality and the general-equilibrium case with observable quality are small.

\subsubsection{The Role of Penalties}

Several European countries have mild or no penalties on illicit drugs' buyers and strong penalties on drugs' sellers, whereas the United States enforce strict penalties on both buyers and sellers. Legal penalties on drug trade obviously affect sellers' costs $K_{S}$ and buyers' costs $K_{B}$ and, thus, in this Section, we use our model to understand how these costs $K_{S}$ and $K_{B}$ affect market outcomes.

Figures 6 and 7 display numerical comparative statics with respect to sellers' cost $K_{S}$ and to buyers' cost $K_{B}$, respectively, in the baseline model of Section 3 with ex-ante unobservable quality. The two figures show that the costs have similar effects on market outcomes. However, the exact mechanism differs in the two cases. Specifically, a larger cost $K_{S}$ decreases the equilibrium mass $S$ of active sellers. Thus, the meeting rate $\alpha(\theta)$ between buyers and sellers decreases. This decrease makes it more difficult for buyers to purchase drugs and, thus, tends to decrease the number of active buyers in the market. Moreover, a lower meeting rate shifts sellers' relative profitability of targeting first-time buyers or loyal buyers. Specifically, a lower meeting rate decreases sellers' incentives to make quick profits and to rip-off buyers by selling $q=0$, thereby increasing the qualities they offer. As a result, the top right panel indicates that the fraction of rip-offs $F_{0}$ decreases, and the bottom left panel displays that the average qualities consumed by first-time buyers increases. This increase 

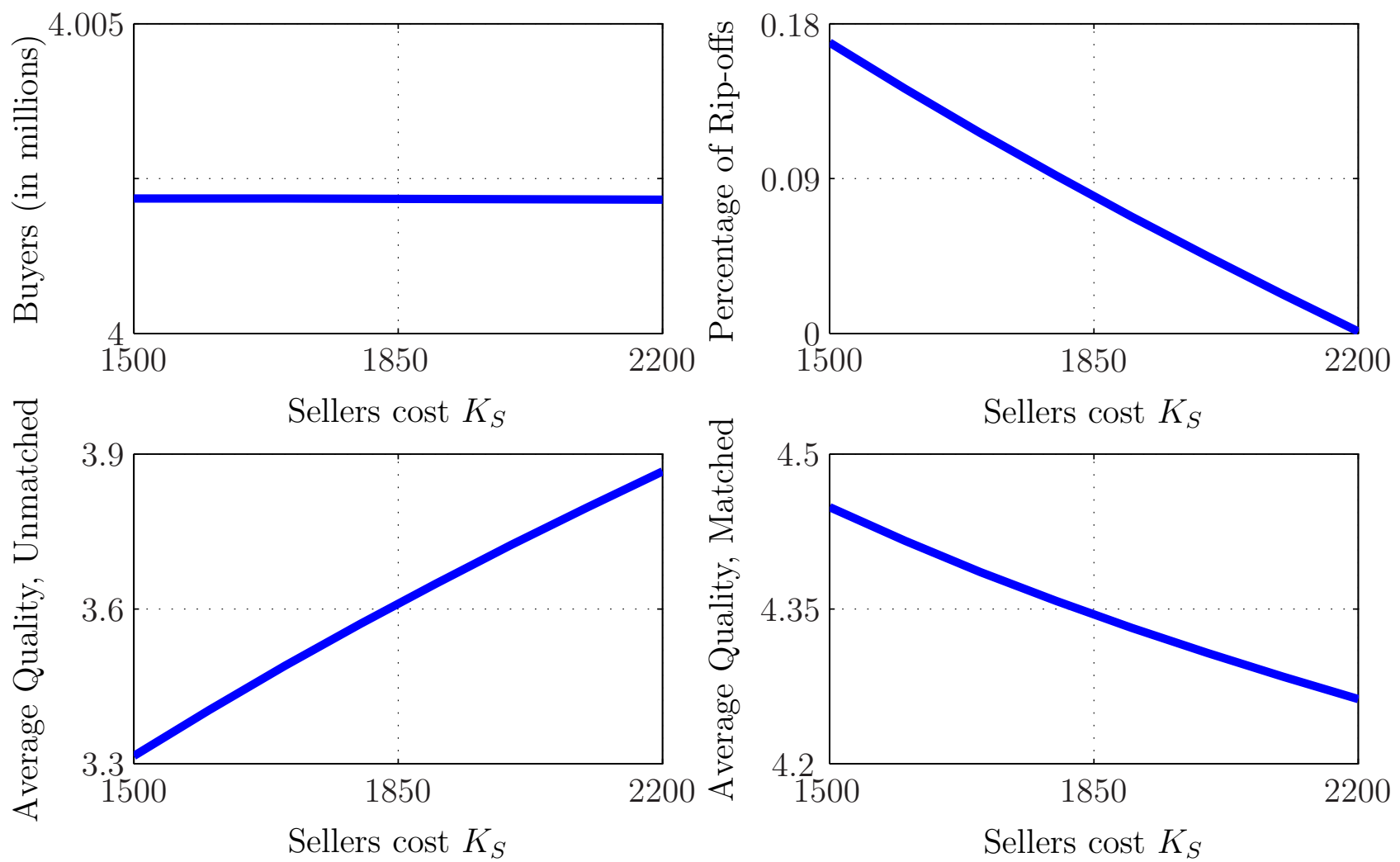

FIG. 6: The effect of sellers' cost $K_{S}$.

in drug quality tends to increase the number of active buyers in the market. As a result of these two opposite forces - i.e., lower meeting rate and higher drug quality - the top left panel shows that the equilibrium number of buyers remains approximately constant. However, the lower meeting rate also implies that it is more difficult for buyers to switch to sellers that offer higher-quality drugs. Hence, the bottom right panel shows that the average quality consumed by matched buyers decreases.

The top left panel of figure 7 indicates that a greater buyers' cost $K_{B}$ decreases the mass of active buyers in the market, thereby increasing buyers' marginal type $z^{*}$. Since the remaining buyers have, on average, a lower reservation value, it becomes more profitable for sellers to try to attract long-term buyers by offering them a low, but positive quality rather than to rip them off by selling $q=0$. Hence, the top right panel shows that the fraction of rip-offs $F_{0}$ decreases and the bottom left panel shows that the average quality consumed by unmatched buyers increases. However, the bottom right panel shows that average quality consumed by matched buyers decreases, as the density of low, but positive, quality just above $\underline{q}$ increases. 

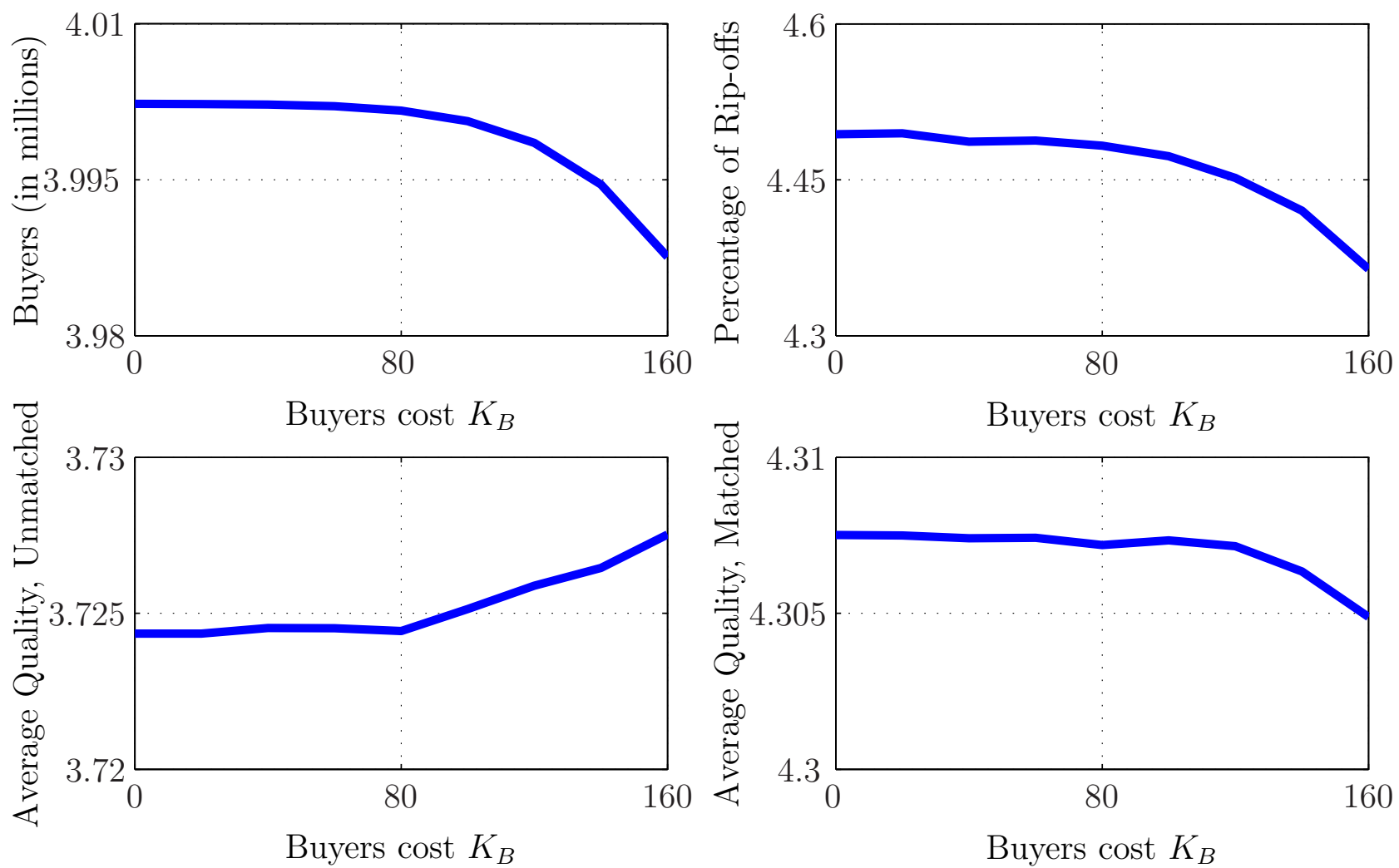

FIG. 7: The effect of buyers' cost $K_{B}$.

\section{Conclusions}

This paper develops a framework to understand illicit drug markets. We focus on two key characteristics of illegal markets: 1) the inability to verify/contract the quality of the good; and 2) penalties on market participants. We estimate the model using data on the U.S. market for powder cocaine. The model fits the data well. Our counterfactual analysis implies that sellers' moral hazard reduces the average and increases the dispersion of drug purity, thereby reducing drug consumption. Moreover, the estimated model implies that increasing penalties may increase the purity and the affordability of drugs traded, because it increases sellers' relative profitability of targeting loyal buyers versus first-time buyers. 


\section{APPENDIX}

\section{A Observable Drug Purity}

In this Appendix, we characterize the market equilibrium when buyers can observe drug quality before purchasing it.

\section{A.1 The Buyers}

Proposition 9 Given $F(\cdot)$ and $S$ :

1. If $\frac{p}{\bar{q}} \geq \bar{z}$ then there is no buyer entry: $B=0$.

2. If $\frac{p}{\bar{q}}<\bar{z}$ then there is a unique buyer type $z^{*} \leq \bar{z}$ such that all buyers with $z>z^{*}$ participate in the market and all buyers with $z \leq z^{*}$ do not.

3. The measure of buyers in the market is $B=\bar{B}\left(1-\tilde{M}\left(z^{*}\right)\right)$ and the distribution of their types in the market is given by

$$
M(z)= \begin{cases}0 & \text { if } z \leq z^{*} \\ \frac{\tilde{M}(z)-\tilde{M}\left(z^{*}\right)}{1-\tilde{M}\left(z^{*}\right)} & \text { if } z \geq z^{*}\end{cases}
$$

4. The marginal buyer type is given by the solution to:

$$
z^{*} \alpha_{B}(\theta) \int_{p / z^{*}}^{\bar{q}}\left(1+\frac{\phi}{r+\delta+\alpha_{B}(\theta)(1-F(q))}\right)(1-F(q)) d q=K_{B}
$$

5. The reservation quality of a type-z buyer who participates in the market is $R_{z}=\frac{p}{z}$ and the distribution of reservation qualities in the market is

$$
H(R)= \begin{cases}0 & \text { if } R \leq \underline{R} \\ \frac{1-M\left(\frac{p}{R}\right)}{1-M\left(z^{*}\right)} & \text { if } R \in[\underline{R}, \bar{R}] \\ 1 & \text { if } R \geq \bar{R}\end{cases}
$$


where $\underline{R}=R_{z}=\frac{p}{z}$ and $\bar{R}=R_{z^{*}}=\frac{p}{z^{*}}$.

A buyer observes the quality offered before purchasing. Therefore, at each state he has to choose whether to consume and whether to become matched with that seller. His value functions are given by:

$$
\begin{aligned}
r \bar{V}_{z} & =\alpha_{B}(\theta) \int_{0}^{\bar{q}}\left(\max \left[z \tilde{q}-p+\max \left[V_{z}(\tilde{q})-\bar{V}_{z}, 0\right], 0\right]\right) d F(\tilde{q}) \\
r V_{z}(q) & =\phi(z q-p)+\alpha_{B}(\theta) \int_{0}^{\bar{q}}\left(\max \left[z \tilde{q}-p+\max \left[V_{z}(\tilde{q})-V_{z}(q), 0\right], 0\right]\right) d F(\tilde{q})+\delta\left(\bar{V}_{z}-V_{z}(q)\right)
\end{aligned}
$$

The reservation quality for consumption is the same regardless of whether the buyer is matched or not and is denoted by $\hat{R}_{z}$. Comparing the static costs and benefits of consumption we have:

$$
\hat{R}_{z}=\frac{p}{z}
$$

When the buyer is matched with a seller who offers $q$, his reservation for matching with a new seller is $q$. When the buyer is unmatched, his reservation is denoted by $R_{z}$. Equating the two value functions delivers the reservation quality for becoming matched:

$$
R_{z}=\hat{R}_{z}=\frac{p}{z}
$$

We can rewrite the value functions as follows:

$$
\begin{aligned}
r \bar{V}_{z} & =\alpha_{B}(\theta) \int_{\frac{p}{z}}^{\bar{q}}\left(z \tilde{q}-p+V_{z}(\tilde{q})-\bar{V}_{z}\right) d F(\tilde{q}) \\
r V_{z}(q) & =\phi(z q-p)+\alpha_{B}(\theta)\left(\int_{\frac{p}{z}}^{\bar{q}}(z \tilde{q}-p) d F(\tilde{q})+\int_{q}^{\bar{q}}\left(V_{z}(\tilde{q})-V_{z}(q)\right) d F(\tilde{q})\right)+\delta\left(\bar{V}_{z}-V_{z}(q)\right)
\end{aligned}
$$

An individual buyer takes as given the actions of sellers $\{F(\cdot), S\}$ and other buyers $(B)$ and decides whether to participate in the market. The actions of other agents are summarized as $\{F(\cdot), \theta\}$. To examine the individual buyer's choice, we write his value of participating $\bar{V}_{z}$ in a more convenient way. 
Using integration by parts the value of being unmatched can be written as:

$$
\begin{aligned}
r \bar{V}_{z} & =\alpha_{B}(\theta)\left(\left.\left(z q-p+V_{z}(q)-\bar{V}_{z}\right) F(q)\right|_{R_{z}} ^{\bar{q}}-\int_{R_{z}}^{\bar{q}}\left(z+V_{z}^{\prime}(q)\right) F(q) d q\right) \\
& =\alpha_{B}(\theta)\left(z \bar{q}-p+V_{z}(\bar{q})-\bar{V}_{z}-F\left(R_{z}\right)\left(z R_{z}-p+V_{z}\left(R_{z}\right)-\bar{V}_{z}\right)-\int_{R_{z}}^{\bar{q}}\left(z+V_{z}^{\prime}(q)\right) F(q) d q\right) \\
& =\alpha_{B}(\theta)\left(z\left(\bar{q}-R_{z}\right)+V_{z}(\bar{q})-V_{z}\left(R_{z}\right)-\int_{\frac{p}{z}}^{\bar{q}}\left(z+V_{z}^{\prime}(q)\right) F(q) d q\right)
\end{aligned}
$$

where we used $R_{z}=\frac{p}{z}$ and $\bar{V}_{z}=V_{z}\left(R_{z}\right)$ in the last equality.

Using the fundamental theorem of calculus:

$$
\begin{aligned}
r \bar{V}_{z} & =\alpha_{B}(\theta)\left(\int_{\frac{p}{z}}^{\bar{q}}\left(z+V_{z}^{\prime}(x)\right) d x-\int_{\frac{p}{z}}^{\bar{q}}\left(z+V_{z}^{\prime}(x)\right) F(x) d x\right) \\
& =\alpha_{B}(\theta) \int_{\frac{p}{z}}^{\bar{q}}\left[z+V_{z}^{\prime}(q)\right](1-F(x)) d x
\end{aligned}
$$

Differentiate the value of being matched with respect to $q$ and rearrange to get:

$$
V_{z}^{\prime}(q)=\frac{\phi z}{r+\delta+\alpha_{B}(\theta)(1-F(q))}
$$

Combining the previous two equations:

$$
r \bar{V}_{z}=z \alpha_{B}(\theta) \int_{\frac{p}{z}}^{\bar{q}}\left(1+\frac{\phi}{r+\delta+\alpha_{B}(\theta)(1-F(x))}\right)(1-F(x)) d x
$$

We now determine whether a buyer of type $z$ participates in the market. Notice that buyers with $z \leq \frac{p}{\bar{q}}$ have no benefit from participating in the market and never enter. For buyers with $z>\frac{p}{\bar{q}}$ we have:

$$
\begin{aligned}
& \lim _{\theta \rightarrow 0} r \bar{V}_{z}=\lim _{\theta \rightarrow 0} \alpha_{B}(\theta) \int_{\frac{p}{z}}^{\bar{q}}(1-F(x)) d x>0 \\
& \lim _{\theta \rightarrow 0} r \bar{V}_{z}=0
\end{aligned}
$$

Therefore, a buyer with $z>\frac{p}{\bar{q}}$ might enter if the arrival rate of new meetings is high enough and does not enter if $z \leq \frac{p}{\bar{q}}$ regardless of $\theta$. As a corollary, if $\bar{z} \leq \frac{p}{\bar{q}}$ then no buyer enters. Furthermore, fixing a buyer's type and increasing $\theta$, i.e. increasing the buyer-seller ratio, 
reduces that buyer's value of participating in the market:

$$
\frac{\partial r \bar{V}_{z}}{\partial \theta}=z \int_{\frac{p}{z}}^{\bar{q}}\left(\alpha_{B}^{\prime}(\theta)+\frac{\alpha_{B}^{\prime}(\theta)(r+\delta)}{\alpha_{B}(\theta)^{2}} \frac{\phi}{\left(\frac{r+\delta}{\alpha_{B}(\theta)}+1-F(x)\right)^{2}}\right)(1-F(x)) d x<0 .
$$

In the limit, if a buyer never meets with sellers, then he does not enter:

$$
\lim _{\theta \rightarrow \infty} r \bar{V}_{z}=0<K_{B}
$$

Therefore, for each buyer of type $z$ with $z>\frac{p}{\hat{q}}$ there is a unique $\theta(z)$ such that he participates if $\theta \leq \theta(z)$ and stays out otherwise.

The value of participating in the market is, unsurprisingly, negative for buyers who receive no utility from consuming and is strictly increasing in a buyer's marginal utility of consumption:

$$
\begin{aligned}
r \bar{V}_{0} & =-\alpha_{B}(\theta) p<0 \\
\frac{\partial r \bar{V}_{z}}{\partial z} & =\alpha_{B}(\theta) \int_{0}^{\bar{q}} x d F(x)+\alpha_{B}(\theta) \int_{R_{z}}^{\bar{q}} \frac{\phi(1-F(x))}{r+\delta+\alpha_{B}(\theta)(1-F(x))} d x+\frac{p}{z^{2}} \frac{\alpha_{B}(\theta) \phi\left(1-F\left(R_{z}\right)\right)}{r+\delta+\alpha_{B}(\theta)\left(1-F\left(R_{z}\right)\right)}>0
\end{aligned}
$$

Taking $\theta$ as given, there is a unique $z(\theta)$ such that a buyer participates if $z \geq z(\theta)$ and does not participate otherwise.

We now prove that $z^{*}$ is unique, taking into account that the number of buyers depends on $z^{*}$ according to $B=\bar{B}\left(1-\tilde{M}\left(z^{*}\right)\right)$. First, note that when $z^{*}=0$ we have $r \bar{V}_{z^{*}}<K_{B}$. Furthermore, when $z^{*}=\bar{z}$ we have $r \bar{V}_{\bar{z}}>K_{B}$, assuming of course that $\bar{z}>\frac{p}{\hat{q}}$, because otherwise no buyers enter.

To prove that the uniqueness of $z^{*}$ we need to show that the value of the marginal type is increasing in his own type. The unmatched value of the marginal buyer depends on $z^{*}$ as follows:

$$
\frac{d r \bar{V}_{z^{*}}}{d z^{*}}=\frac{\partial r \bar{V}_{z^{*}}}{\partial z^{*}}+\frac{\partial r \bar{V}_{z^{*}}}{\partial \theta}\left(-\bar{B} \tilde{M}^{\prime}\left(z^{*}\right)\right)>0
$$

Therefore, there is a unique $z^{*}$ such that the unmatched value of the marginal buyer is exactly equal to $K_{B}$ and it is defined by equation (1). This completes the proof of Proposition 2, parts 2,3 and 4 . 
Finally, let $z(R)$ denote the buyer type whose reservation quality is equal to $R$. Rearranging equation (4) we have:

$$
z(R)=\frac{p}{R}
$$

Furthermore, note that $R_{z(R)}=R$ and $z \leq z(R) \Leftrightarrow R_{z} \geq R$. Given $z^{*}$, the equilibrium distribution of reservation qualities mirrors the distribution of marginal utilities according to Proposition 2, part 5.

This completes the characterization of buyers' behavior.

\section{A.2 The Sellers}

We derive the sellers' profits and describe their actions, taking as given the measure of buyers who participate $B$ and the distribution of reservation qualities $H(\cdot)$. The distribution of buyer types does not affect sellers over and above the distribution of reservation qualities.

A measure $S$ of sellers participate in the market, which is determined through free entry. Each seller draws the marginal cost $c$ of providing a unit of quality from some distribution $D(\cdot)$. The problem of a seller of type $c$ is to choose a level of quality $\hat{q}(c)$ that maximizes his steady state profits. Steady state profits have two components: the margin per transaction and the steady state flow of transactions. The profit margin from each transaction is equal to $p-c q$. The flow of transactions is $t(q)=t_{N}(q)+t_{L}(q)$ where $t_{N}(q)$ refers to new buyers and $t_{L}(q)$ refers to loyal buyers. Steady state profits ar:

$$
\pi_{c}(q)=(p-c q)\left(t_{N}(q)+t_{L}(q)\right)
$$

We first derive some necessary conditions on the distribution of offered qualities.

Lemma 10 In equilibrium, the quality distribution F:

1. has support on a subset of $[\underline{q}, \bar{q}]$,

2. $\underline{q} \in[\underline{R}, \bar{R}]$,

3. is continuous on $[0, \bar{q}]$.

Proof. For $q \in[0, \underline{R})$ we have $t(q)=0$ and therefore $q \geq \underline{q}$ for some $\underline{q} \geq \underline{R}$. If $\underline{q}>\bar{R}$ then $t(q)=t(\bar{R})$ for $q \in[\bar{R}, \underline{q}]$ which implies that $\pi_{c}(\bar{R})>\pi_{c}(q)$ for $q \in(\bar{R}, \underline{q}]$. Therefore, $\underline{q} \leq \bar{R}$. 
The previous point proves that $F$ is constant (and hence continuous) on $[0, \underline{q}]$. Standard arguments (as in Burdett-Mortensen) prove continuity on $[\underline{q}, \bar{q}]$.

In the following sections we characterize the flow of transactions for any $F$ that satisfies the previous Lemma and then we characterize the seller's optimal quality choice $\hat{q}(c)$.

\section{A.2.1 Characterization of profits}

We take $H(\cdot), F(\cdot)$ and $\theta$ as given and calculate the steady state profits that a type- $c$ seller would enjoy for any quality $q$. The main result is summarized in the next proposition.

Proposition 11 The steady state profits of a seller of type $c$ who offers quality $q$ are:

$$
\pi_{c}(q)=\alpha_{B}(\theta) \theta H(q)(p-c q)\left(1+\frac{\phi \delta}{\left(\delta+\alpha_{B}(\theta)(1-F(q))\right)^{2}}\right), \quad q \geq \underline{q}
$$

To determine profits, we need to first determine the flow of a seller's transactions as a function of the quality he offers. The rate at which an individual seller transacts with a new buyer equals the meeting rate times the probability the seller's quality is above the buyer's reservation:

$$
t_{N}(q)=\alpha_{S}(\theta) H(q)=\theta \alpha_{B}(\theta) H(q)
$$

The flow of transactions from loyal buyers is given by:

$$
t_{L}(q)=\phi l(q)
$$

where $l(q)$ is the steady steady number of loyal buyers of a seller offering $q$.

The number of loyal buyers per seller offering $q$ is given by:

$$
l(q)=\frac{(B-\bar{n}) G^{\prime}(q)}{S F^{\prime}(q)}
$$

where $\bar{n}$ is the number of unmatched buyers, $(B-\bar{n}) G^{\prime}(q)$ is the number of buyers who are matched with a seller offering $q$ and $S F^{\prime}(q)$ is the number of sellers offering quality $q$.

We determine the number of unmatched buyers and their type distribution. In steady state, the flow of buyers from the unmatched to the matched state must equal the flow out 
of the matched state and into the unmatched state. Let $n(R)$ denote the number of buyers who are unmatched and whose type is less than $R$. The total number of unmatched buyers is therefore given by $n(\bar{R}) \equiv \bar{n}$.

An unmatched buyer of type $R$ becomes matched after transacting with a seller who offers above-reservation quality which occurs at rate $\alpha_{B}(\theta)(1-F(R))$. A matched buyer exits the matched state when his match is exogenously destroyed which occurs at rate $\delta$. As a result, in steady state the following holds:

$$
n^{\prime}(R) \alpha_{B}(\theta)(1-(F(R)))=\delta\left(B H^{\prime}(R)-n^{\prime}(R)\right) \Rightarrow n^{\prime}(R)=\frac{\delta B H^{\prime}(R)}{\delta+\alpha_{B}(\theta)(1-F(R))}
$$

Alternatively, this can be written as:

$$
n(R)=\int_{\underline{R}}^{R} \frac{B \delta}{\delta+\alpha_{B}(\theta)(1-F(x))} d H(x)
$$

Therefore, we have:

$$
\begin{aligned}
\bar{n} & =\int_{\underline{R}}^{\bar{R}} \frac{B \delta}{\delta+\alpha_{B}(\theta)(1-F(x))} d H(x) \\
B-\bar{n} & =B\left(1-\int_{\underline{R}}^{\bar{R}} \frac{\delta}{\delta+\alpha_{B}(\theta)(1-F(x))} d H(x)\right) \\
& =\int_{\underline{R}}^{\bar{R}} \frac{B \alpha_{B}(\theta)(1-F(x))}{\delta+\alpha_{B}(\theta)(1-F(x))} d H(x)
\end{aligned}
$$

We now characterize $G(\cdot)$. The mass of matched buyers receiving quality up to $q$ is given by $(B-\bar{n}) G(q)$. An unmatched type- $R$ buyer flows into this group if $R \leq q$ and he samples a seller who offers quality less than $q$, which occurs at rate $\alpha_{B}(\theta)(F(q)-F(R))$. A buyer flows out of this group if the match is exogenously destroyed or if he samples a new seller whose quality if greater than $q$, which occurs at rate $\delta+\alpha_{B}(\theta)(1-F(q))$. Equating these 
flows yields

$$
\begin{aligned}
\alpha_{B}(\theta) \int_{\underline{R}}^{q}(F(q)-F(R)) d n(R) & =(B-\bar{n}) G(q)\left(\delta+\alpha_{B}(\theta)(1-F(q))\right) \\
\Rightarrow(B-\bar{n}) G(q) & =\frac{\alpha_{B}(\theta) \int_{\underline{R}}^{q}(F(q)-F(x)) d n(x)}{\delta+\alpha_{B}(\theta)(1-F(q))} \\
& =\frac{\alpha_{B}(\theta) B \delta \int_{\underline{R}}^{q} \frac{F(q)-F(x)}{\delta+\alpha_{B}(\theta)(1-F(x))} d H(x)}{\delta+\alpha_{B}(\theta)(1-F(q))}
\end{aligned}
$$

Some algebra leads to:

$$
(B-\bar{n}) G^{\prime}(q)=\frac{\alpha_{B}(\theta) B \delta F^{\prime}(q) H(q)}{\left(\delta+\alpha_{B}(\theta)(1-F(q))^{2}\right.}
$$

which implies that the flow of transactions from loyal buyers is:

$$
t_{L}(q)=\frac{\phi \alpha_{B}(\theta) \theta \delta H(q)}{\left(\delta+\alpha_{B}(\theta)(1-F(q))\right)^{2}}
$$

Combining results completes the proof of the Proposition.

\section{A.2.2 The sellers' optimal quality choice}

We now characterize the distribution of offered qualities, $F(\cdot)$ and the number of sellers who enter the market taking as given the number of buyers $B$ and the distribution of their reservation values $H(\cdot)$.

Lemma 12 Consider sellers 1 and 2 and denote their actions by $q_{1}$ and $q_{2}$. We have $c_{1}>$ $c_{2} \Rightarrow q_{2}>q_{1}$.

Proof. The proof is by contradiction. Suppose that $c_{1}>c_{2}$ and $q_{2} \leq q_{1}$. Recall that profits are given by $\pi_{c}(q)=(p-c q) t(q)$.

Seller 1 chose quality $q_{1}$ over $q_{2}$. Therefore:

$$
\begin{aligned}
\left(p-c_{1} q_{1}\right) t\left(q_{1}\right) & \geq\left(p-c_{1} q_{2}\right) t\left(q_{2}\right) \\
\Rightarrow p\left(t\left(q_{1}\right)-t\left(q_{2}\right)\right) & >c_{1}\left(t\left(q_{1}\right) q_{1}-t\left(q_{2}\right) q_{2}\right) \\
\Rightarrow p\left(t\left(q_{1}\right)-t\left(q_{2}\right)\right) & >c_{2}\left(t\left(q_{1}\right) q_{1}-t\left(q_{2}\right) q_{2}\right)
\end{aligned}
$$


where the strict inequality results from $c_{1}>c_{2}$.

Seller 2 chose quality $q_{2}$ over $q_{1}$. Therefore:

$$
\left(p-c_{2} q_{2}\right) t\left(q_{2}\right) \geq\left(p-c_{2} q_{1}\right) t\left(q_{1}\right) \Rightarrow p\left(t\left(q_{1}\right)-t\left(q_{2}\right)\right) \leq c_{2}\left(t\left(q_{1}\right) q_{1}-t\left(q_{2}\right) q_{2}\right)
$$

Therefore, $q_{1}=q_{2}$ is the only possibility that satisfies the above inequalities.

Now consider any seller 3 with $c_{1}>c_{3}>c_{2}$. Such a seller exists because the support of $D(\cdot)$ is connected. If $q_{1}=q_{2}=\tilde{q}$ then the analysis above means that $q_{3}=\tilde{q}$ as well. In that case, there is a mass of sellers offering $\tilde{q}$ equal to $D\left(c_{1}\right)-D\left(c_{2}\right)$ which is inconsistent with equilibrium (see Lemma 10).

One corollary of the previous Lemma is that $F(\hat{q}(c))=1-D(c)$.

We now determine sellers' optimal $\hat{q}(c)$.

Proposition 13 Given $H(\cdot)$ and $\theta$ the optimal quality choice for sellers of type $c<c^{*}$ is given by the solution to the differential equation

$$
\hat{q}^{\prime}(c)=\frac{2(p-c \hat{q}(c)) \phi \delta \alpha_{B}(\theta) D^{\prime}(c)}{\left[\frac{H^{\prime}(\hat{q}(c))}{H(\hat{q}(c))}(p-c \hat{q}(c))-c\right]\left(\delta+\alpha_{B}(\theta) D(c)\right)\left[\left(\delta+\alpha_{B}(\theta) D(c)\right)^{2}+\phi \delta\right]}
$$

where $\underline{q}\left(c^{*}\right)$ is the initial condition, determined by the solution of

$$
\max _{q} \pi\left(c^{*}\right)=\max \alpha_{B}(\theta) \theta H(\underline{q})\left(p-c^{*} \underline{q}\right)\left(1+\frac{\phi \delta}{\left.\left(\delta+\alpha_{B}(\theta)\right)\right)^{2}}\right) .
$$

The distribution of qualities is:

$$
F(q)=1-D\left(\hat{q}^{-1}(q)\right)
$$

To characterize the function of optimal quality offer $\hat{q}(c)$ we rewrite the profits of a type- $c$ seller as if he decides which other type $c^{\prime}$ to imitate rather than which quality to offer. In other words, his profits from offering some quality $q^{\prime}$ are written in terms of imitating type $c^{\prime}$ who offers quality $q^{\prime}=\hat{q}\left(c^{\prime}\right)$. We have:

$$
\pi_{c}\left(c^{\prime}\right)=\alpha_{B}(\theta) \theta H\left(\hat{q}\left(c^{\prime}\right)\right)\left(p-c \hat{q}\left(c^{\prime}\right)\right)\left(1+\frac{\phi \delta}{\left(\delta+\alpha_{B}(\theta) D\left(c^{\prime}\right)\right)^{2}}\right)
$$


The advantage of formulating the choice in terms of $c^{\prime}$ rather than $q^{\prime}$ is that the term in the denominator depends on the exogenous type distribution $D(\cdot)$ rather than the endogenous quality distribution $F(\cdot)$. The quality distribution will be recovered once $\hat{q}(c)$ is constructed.

Differentiate profits with respect to $c^{\prime}$

$$
\begin{aligned}
\pi_{c}^{\prime}(\hat{c} ; c)= & \alpha_{B}(\theta) \theta\left(H^{\prime}\left(\hat{q}\left(c^{\prime}\right)\right) \hat{q}^{\prime}(c)(p-c \hat{q}(c))\left(1+\frac{\phi \delta}{\left(\delta+\alpha_{B}(\theta) D\left(c^{\prime}\right)\right)^{2}}\right)\right. \\
& -c \hat{q}^{\prime}(c) H(\hat{q}(c))\left(1+\frac{\phi \delta}{\left(\delta+\alpha_{B}(\theta) D\left(c^{\prime}\right)\right)^{2}}\right) \\
& \left.-H(\hat{q})(p-c \hat{q}(c)) \frac{2 \phi \delta \alpha_{B}(\theta) D^{\prime}\left(c^{\prime}\right)}{\left(\delta+\alpha_{B}(\theta) D\left(c^{\prime}\right)\right)^{3}}\right)
\end{aligned}
$$

By construction, profits are maximized when $\hat{c}=c$ and we can therefore set the derivative to zero and rearrange to arrive at

$$
\hat{q}^{\prime}(c)=\frac{2(p-c \hat{q}(c)) \phi \delta \alpha_{B}(\theta) D^{\prime}(c)}{\left[\frac{H^{\prime}(\hat{q}(c))}{H(\hat{q}(c))}(p-c \hat{q}(c))-c\right]\left(\delta+\alpha_{B}(\theta) D(c)\right)\left[\left(\delta+\alpha_{B}(\theta) D(c)\right)^{2}+\phi \delta\right]}
$$

which determines $\hat{q}(c)$.

Notice that all terms of $\hat{q}^{\prime}(c)$ are always positive except for the first term of the denominator. Therefore the sign of $\hat{q}^{\prime}(c)$ is the same at the sign of $T(c)$ where

$$
T(c)=\frac{H^{\prime}(\hat{q}(c))}{H(\hat{q}(c))}(p-c \hat{q}(c))-c
$$

Define $\underline{c}$ such that $\hat{q}(\underline{c})=\bar{R}$ and notice that $c<\underline{c} \Rightarrow \hat{q}(c)>\bar{R} \Rightarrow H^{\prime}(\hat{q}(c))=0$ which means that $T(c)<0$. For $c>\underline{c}$, we have that $T(c)=0$ for $q=\underline{q}$ where $\hat{q}\left(c^{*}\right)=\underline{q}$. Therefore, for $c \in\left(\underline{c}, c^{*}\right)$ we have $T(c)<0$ and $\hat{q}^{\prime}(c)<0$.

Having fully characterized $F(\cdot)$, we turn to determining the number of sellers $S$ who choose to enter the market.

Proposition 14 Given $H(\cdot)$ and $B$ there is a unique $S$ such that $\Pi=K_{S}$.

The key for this proposition is that profits for every type of seller are increasing in $\theta$ :

$$
\frac{d \pi_{c}(q)}{d \theta}=\frac{\partial \pi_{c}(q)}{\partial \theta}+\frac{\partial \pi_{c}(q)}{\partial q} \frac{d q}{d \theta}
$$


The first term is positive. The second terms is zero by the envelope theorem. Furthermore:

$$
\begin{aligned}
& \lim _{\theta \rightarrow 0} \pi_{c}(q)=0 \\
& \lim _{\theta \rightarrow \infty} \pi_{c}(q)>K_{S}
\end{aligned}
$$

which proves the Proposition.

This completes the characterization of sellers' behavior.

\section{References}

[1] Arkes, J., R.L. Pacula, S. Paddock, J. Caulkins, and P. Reuter. (2004). "Technical Report for the Price and Purity of Illicit Drugs Through 2003." Office of National Drug Control Policy. November.

[2] Arkes, J., S. M. Paddock, J. P. Caulkins, and P. Reuter (2008). "Why the DEA STRIDE Data are Still Useful for Understanding Drug Markets." NBER Working Paper 14224, August 2008.

[3] Bontemps, C., J.-M. Robin and G. Van der Berg (1998). "An Empirical Equilibrium Job Search Model with Search on the Job and Heterogeneous Workers and Firms." International Economic Review, Vol. 40, No. 4, pp. 1039-1074.

[4] Burdett, K. and D. T. Mortensen (1998). "Wage Differentials, Employer Size, and Unemployment." International Economic Review, Vol. 39, pp. 257-273.

[5] Davies, R. (2010). "Mandatory Minimum Sentencing, Drug Purity and Overdose Rates." The Economic and Social Review, Vol. 41, pp. 429-457.

[6] Freeborn, B. (2009). "Arrest Avoidance: Law Enforcement and the Price of Cocaine." Journal of Law and Economics, Vol. 52, pp. 19-40.

[7] Galenianos, M., R. Liccardo Pacula and N. Persico (2012). "A Search-Theoretic Model of the Retail Market for Illicit Drugs. " Review of Economic Studies, Vol. 79, pp. 12391269. 
[8] Horowitz, J. (2001). "Should the DEA's Stride Data Be Used for Economic Analyses of Markets for Illegal Drugs?" Journal of the American Statistical Association, Vol. 96.

[9] Kuziemko, I. and S. Levitt (2004). "An empirical analysis of imprisoning drug offenders." Journal of Public Economics, Vol. 88, pp. 2043-2066.

[10] Levitt, S. and S. Venkatesh (2000). "An Economic Analysis of a Drug-Selling Gang's Finances." Quarterly Journal of Economics, Vol. 115, pp. 755-789.

[11] Reuter, P. and J. Caulkins (2004). "Illegal Lemons: Price Dispersion in the Cocaine and Heroin Markets." UN Bulletin on Narcotics. 